\title{
Edge offset meshes in Laguerre geometry
}

Helmut Pottmann, Philipp Grohs, and Bernd Blaschitz

$$
\text { Project Area(s): }
$$

Industrielle Geometrie - Hoehere Geometrie

Industrielle Geometrie - Algorithmische Differentialgeometrie

Institut für Geometrie 


\title{
Edge offset meshes in Laguerre geometry
}

\begin{abstract}
A mesh $\mathcal{M}$ with planar faces is called an edge offset (EO) mesh if there exists a combinatorially equivalent mesh $\mathcal{M}^{d}$ such that corresponding edges of $\mathcal{M}$ and $\mathcal{M}^{d}$ lie on parallel lines of constant distance $d$. The edges emanating from a vertex of $\mathcal{M}$ lie on a right circular cone. Viewing $\mathcal{M}$ as set of these vertex cones, we show that the image of $\mathcal{M}$ under any Laguerre transformation is again an EO mesh. As a generalization of this result, it is proved that the cyclographic mapping transforms any EO mesh in a hyperplane of Minkowksi 4-space into a pair of Euclidean EO meshes. This result leads to a derivation of EO meshes which are discrete versions of Laguerre minimal surfaces. Laguerre minimal EO meshes can also be constructed directly from certain pairs of Koebe meshes with help of a discrete Laguerre geometric counterpart of the classical Christoffel duality.
\end{abstract}

\section{Introduction}

Recent research revealed a close connection between discrete differential geometry and the design and construction of architectural freeform structures $\left[\mathrm{G}^{*} 02, \mathrm{LPW}^{*} 06, \mathrm{PAHK} 07, \mathrm{PCW} 07, \mathrm{PLW}^{*} 07, \mathrm{Sch} 03\right]$. It turned out that practical requirements make certain discrete surface representations such as meshes with planar faces and offset properties very attractive for architectural applications. In fact, the architectural application led to the formulation of some new concepts in discrete differential geometry.

An important class of discrete surfaces for architectural design are conical meshes [LPW 06 ]. A conical mesh $\mathcal{M}$ possesses planar faces. At each vertex of $\mathcal{M}$, the planes of adjacent faces are tangent to a right circular cone. This implies that a conical mesh possesses conical offset meshes $\mathcal{M}^{d}$ which

Helmut Pottmann, Philipp Grohs and Bernhard Blaschitz

Institut für Diskrete Mathematik und Geometrie, Technische Universität Wien

Wiedner Hauptstr. 8-10/104, A 1040 Wien, Austria

E-mail: \{pottmann,grohs,blaschitz\}@geometrie.tuwien.ac.at 


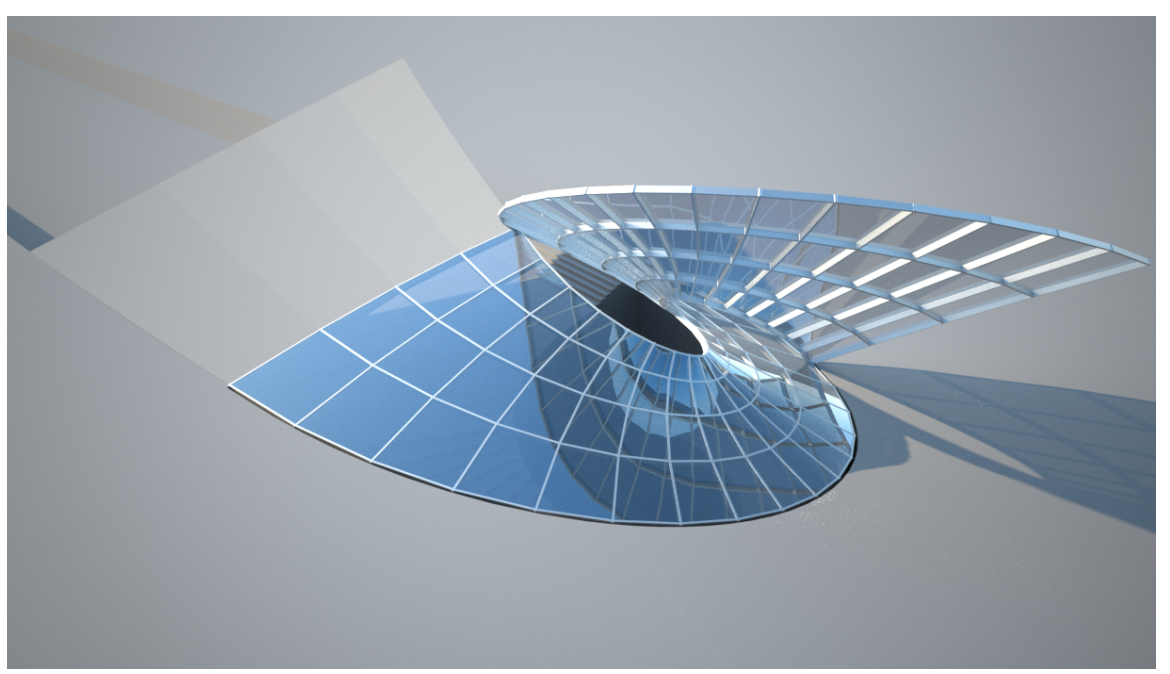

Fig. 1 This design is based on an Enneper L-minimal surface of the spherical type modelled as an edge offset mesh.

have the same combinatorics as $\mathcal{M}$ and whose face planes possess constant distance $d$ to the corresponding face plane of the base mesh $\mathcal{M}$. Orienting the face planes and viewing a conical mesh as set of oriented face planes, it is easy to see that a Laguerre transformation maps a conical mesh to a conical mesh. The offsetting operation is a special instance of a Laguerre transformation, which is in agreement with the fact that the offset meshes are conical. One is mainly interested in quadrilateral conical meshes; these turn out to be discrete versions of the network of principal curvature lines on a surface. Conical triangle meshes are trivial since all face planes are tangent to the same sphere; a hexagonal mesh or more generally a mesh with planar faces and vertices of valence 3 is always conical.

There are also meshes which possess offsets such that corresponding vertices are at constant distance. The quad meshes of that type are the circular meshes which - as sets of vertices - are invariant under Möbius transformations [PW07].

The present paper focusses on the third type of meshes with planar faces and exact offset meshes. Again, the offsets $\mathcal{M}^{d}$ have face planes and edges which are parallel to their correspondents in $\mathcal{M}$, but now the constant distance is realized between corresponding parallel edges (in fact, straight lines carrying these edges). These edge offset (EO) meshes have recently been introduced in [PLW*07], motivated by their capability of forming the best possible nodes in a beam layout for a steel/glass or similar architectural construction. It has been observed that - when viewed properly - these meshes are also invariant under Laguerre transformations. This invariance property is much less obvious than in the case of conical meshes. In fact, we cannot view the mesh as set of planes, edges or vertices to obtain this invariance. One must view an edge offset mesh as a set of certain right circular cones attached to its vertices. Since the proof of the Laguerre 
invariance of EO meshes has not been provided in [PLW*07], it will be given now. Along with this proof, we will get additional insight into the relations between Laguerre geometry and EO meshes. These relations will lead us to further results, the most important of them being a derivation of EO meshes which may be seen as discrete Laguerre minimal surfaces.

\subsection{Previous work}

Discrete differential geometry is an active research area with a variety of applications in various branches of geometric computing and in other theories such as integrable systems. The recently published monograph [BS08] provides an excellent account of this rapidly expanding field. Edge offset meshes have been introduced in [PLW*07]. A central result states that the edges of an EO mesh are parallel to the corresponding edges of a combinatorially equivalent mesh all whose edges are tangent to a sphere. The latter meshes are called Koebe meshes. They enjoy remarkable geometric properties and may be computed as minimizers of a convex function [Sch97, BS04]. Via a discrete counterpart to the Christoffel transformation, Koebe meshes can be transformed into discrete minimal surfaces [BHS06]. In the present paper we will also investigate EO meshes, Koebe meshes and discrete minimal surfaces in pseudo-Euclidean and isotropic 3-space, respectively. Various types of meshes in isotropic 3-space have recently been investigated in [PL07]. In view of the isomorphism between Euclidean Laguerre geometry and isotropic Möbius geometry, that work exhibits close connections to the present study.

Laguerre geometry is the geometry of oriented planes and spheres in Euclidean 3-space [Bla29, Cec92]. Its recent application in the study of discrete surfaces with applications to multi-layer freeform structures for architecture $\left[\mathrm{BS} 06, \mathrm{LPW}^{*} 06, \mathrm{PLW}^{*} 07, \mathrm{WP} 08\right]$ is one of the motivations for the present work. The role of EO meshes in Laguerre geometry is further supported by the observation made in [PLW*07] that quadrilateral EO meshes are discrete counterparts of Blaschke's Laguerre-isothermic surfaces [Bla29]. Later we will derive all EO meshes which can be viewed as discrete Laguerreminimal surfaces. L-minimal surfaces are defined as the minimizers of the energy $\int\left(H^{2}-K\right) / K d A$. Many results are found in the work of Blaschke [Bla24, Bla25, Bla29] and his student König [Kön26, Kön28]. More recent contributions are due to Musso and Nicolodi [MN95, MN96] and Palmer [Pal99]. For the computation of L-minimal surfaces one can exploit the isotropic model of Laguerre geometry, where an L-miminal surface appears as the graph surface of a biharmonic function [PGM08].

\subsection{Contributions and overview}

The contributions of the present paper are as follows:

1. A brief overview of Laguerre geometry and the cyclographic mapping along with some details needed in later sections are provided in Section 2. 
2. Section 3 shows how edge offset meshes can be seen as objects of Laguerre geometry and proves their invariance under Laguerre transformations.

3. Generalizing the L-invariance, Section 4 studies EO meshes $\mathcal{M}$ in hyperplanes $H$ of Minkowski 4-space. It is shown that the cyclographic image of such a mesh $\mathcal{M} \subset H$ consists of a pair of Euclidean EO meshes $\mathcal{M}_{1}, \mathcal{M}_{2}$. Particular emphasis is put on the special case where $\mathcal{M}$ is tangent to a sphere; i.e., $\mathcal{M}$ is a Euclidean, pseudo-Euclidean or isotropic Koebe mesh $\mathcal{S}$. The cyclographic mapping yields remarkable pairs $\mathcal{S}_{1}, \mathcal{S}_{2}$ of Euclidean Koebe meshes.

4. A Laguerre geometric version of the discrete Christoffel duality is used in Section 5 to derive from Koebe pairs $\mathcal{S}_{1}, \mathcal{S}_{2}$ those EO meshes which are discrete Laguerre-minimal surfaces.

5. Finally, Section 6 addresses computational issues and illustrates a few basic examples of L-minimal EO meshes. We also point to open problems in this field.

\section{Laguerre geometry}

Laguerre geometry is the geometry of oriented planes and oriented spheres in Euclidean $E^{3}$. For a thorough treatment of this classical geometry, we refer to the monographs by [Bla29] and [Cec92]. An introduction with applications in CAGD has been provided by [PP98].

We address here only a few basics which are essential for the understanding of the present paper: We may write an oriented (or.) plane $P$ in Hesse normal form $\mathbf{n}^{T} \cdot \mathbf{x}+h=0$, where the unit normal vector $\mathbf{n}$ defines the orientation; $\mathbf{n}^{T} \cdot \mathbf{x}+h$ is the signed distance of the point $\mathbf{x}$ to $P$. An oriented sphere $S$, with center $\mathbf{m}$ and signed radius $R$, is tangent to an oriented plane $P$ if the signed distance of $\mathbf{m}$ to $P$ equals $R$, i.e., $\mathbf{n}^{T} \cdot \mathbf{m}+h=R$. Points are viewed as or. spheres with radius zero.

Laguerre transformations. A Laguerre transformation (L-transformation) is a mapping which is bijective on the sets of or. planes and or. spheres, respectively, and keeps plane/sphere tangency.

L-transformations are more easily understood if we use the so-called cyclographic model of Laguerre geometry. There, an or. sphere $S$ is represented as point $\mathbf{s}:=(\mathbf{m}, R) \in \mathbb{R}^{4}$. An oriented plane $P$ in $E^{3}$ may be interpreted as set of all or. spheres which are tangent to $P$. Mapping $P$ via this set of spheres into $\mathbb{R}^{4}$, one finds a hyperplane in $\mathbb{R}^{4}$ which is parallel to a tangent hyperplane of the cone $x_{1}^{2}+x_{2}^{2}+x_{3}^{2}-x_{4}^{2}=0$. In the cyclographic model, an L-transformation is seen as a special affine map (Lorentz transformation)

$$
\mathbf{s}^{\prime}=\mathbf{a}+L \cdot \mathbf{s},
$$

where $L$ denotes the matrix of a linear map $\mathbb{R}^{4} \rightarrow \mathbb{R}^{4}$ which preserves the inner product

$$
\langle\mathbf{x}, \mathbf{y}\rangle:=x_{1} y_{1}+x_{2} y_{2}+x_{3} y_{3}-x_{4} y_{4}
$$


With the diagonal matrix $D:=\operatorname{diag}(1,1,1,-1)$ we have $\langle\mathbf{x}, \mathbf{y}\rangle=\mathbf{x}^{T} \cdot D \cdot \mathbf{y}$, and the condition on $L$ reads

$$
L^{T} \cdot D \cdot L=D .
$$

$\mathbb{R}^{4}$ equipped with the inner product (2) is also known as pseudo-Euclidean 4 -space or Minkowski space and denoted by $\mathbb{R}^{3,1}$. This geometry is also known from special relativity and some notations are taken from this important link: A vector $\mathbf{v}$ is called space-like if $\langle\mathbf{v}, \mathbf{v}\rangle>0$, time-like if $\langle\mathbf{v}, \mathbf{v}\rangle<0$ and light-like if $\langle\mathbf{v}, \mathbf{v}\rangle=0$. If two vectors $\mathbf{a}, \mathbf{b}$ are Minkowski-orthogonal, $\langle\mathbf{a}, \mathbf{b}\rangle=0$, and one of them is time-like, then the other one must be spacelike. L-transformations do not change the type of vectors.

Let us return to the standard model in $E^{3}$. A pencil of parallel or. planes has the same normal vector $\mathbf{n}$ (image point on the "Gaussian" sphere $S^{2}$ ). An L-transformation keeps the parallelity of or. planes and induces a Möbius transformation of the Gaussian sphere $S^{2}$. Note that an L-transformation does in general not preserve points, since those are seen as special spheres and may be mapped to other spheres. A simple example of an L-transformation is the offset operation (given by (1) with $L$ as identity matrix and $\mathbf{a}=(0,0,0, d))$, which adds a constant $d$ to the radius of each sphere.

Geometry in hyperplanes of $\mathbb{R}^{3,1}$. There are three types of hyperplanes in $\mathbb{R}^{3,1}$, depending on the type of metric which is induced by the inner product (2):

(i) In a Euclidean hyperplane $H$ all vectors $\mathbf{v} \in H$ are space-like, i.e. the restriction of the inner product (2) to vectors in $H$ is positive definite und thus defines a Euclidean metric in $H$. Writing the equation of $H$ as $\langle\mathbf{u}, \mathbf{x}\rangle+u_{0}=0$, the vectors $\mathbf{v} \in H$ satisfy $\langle\mathbf{u}, \mathbf{v}\rangle=0$. It follows that we have $\langle\mathbf{v}, \mathbf{v}\rangle>0$ for all $\mathbf{v}$, i.e., $H$ is space-like, if and only if $\langle\mathbf{u}, \mathbf{u}\rangle\langle 0$. Via a suitable L-transformation, a Euclidean hyperplane can be mapped into the hyperplane $x_{4}=0$ which represents our Euclidean space $E^{3}$ in which the standard model of Laguerre geometry is situated.

(ii) A pseudo-Euclidean hyperplane $H$ contains both space-like and time-like vectors. Its equation reads $\langle\mathbf{u}, \mathbf{x}\rangle+u_{0}=0$ with $\langle\mathbf{u}, \mathbf{u}\rangle>0$. An appropriate L-transformation maps $H$ to $x_{1}=0$.

(iii) An isotropic hyperplane $H$ contains a single direction of light-like vectors and all other vectors are space-like. It is characterized by an equation $\langle\mathbf{u}, \mathbf{x}\rangle+u_{0}=0$ with $\langle\mathbf{u}, \mathbf{u}\rangle=0$. Via an $L$-transformation, we can map $H$ to $x_{1}-x_{4}=0$.

Cyclographic mapping. A point $\mathbf{x}=\left(x_{1}, \ldots, x_{4}\right) \in \mathbb{R}^{3,1}$ corresponds to the oriented sphere in $E^{3}$ with midpoint $\left(x_{1}, x_{2}, x_{3}\right)$ and signed radius $x_{4}$. The mapping $\zeta$ which maps points $\mathbf{x} \in \mathbb{R}^{3,1}$ to or. spheres $\zeta(\mathbf{x}) \subset E^{3}$ is called cyclographic mapping.

Let us give some examples of cyclographic images. A straight line $g \subset$ $\mathbb{R}^{3,1}$ with a space-like direction vector is mapped to a set $\zeta(g)$ of oriented spheres whose envelope is in general an oriented cone of revolution; this envelope may also be a cylinder of revolution or just a straight line. If the 
line $g$ is light-like, the image spheres of its points touch an oriented plane of $E^{3}$ at a point $\mathbf{p}$ (the intersection point $g \cap E^{3}$ ). For a straight line $g$ with a space-like direction vector the cyclographic image spheres do not possess a real envelope.

For later use, we also discuss the image spheres of points in a hyperplane $H \subset \mathbb{R}^{3,1}$. Applying a translation parallel to $E^{3}$ we may achieve that $H$ passes through the origin and thus we can write $H: u_{1} x_{1}+u_{2} x_{2}+u_{3} x_{3}-u_{4} x_{4}=0$. Its intersection with $E^{3}: x_{4}=0$ is the plane

$$
p_{H}: u_{1} x_{1}+u_{2} x_{2}+u_{3} x_{3}=0 .
$$

The image sphere $\zeta(\mathbf{p})$ of a point $\mathbf{p}$ in $H$ has midpoint $\left(p_{1}, p_{2}, p_{3}\right)$ which lies at oriented distance

$$
d=\frac{u_{1} p_{1}+u_{2} p_{2}+u_{3} p_{3}}{\sqrt{u_{1}^{2}+u_{2}^{2}+u_{3}^{2}}}=\frac{u_{4} p_{4}}{\sqrt{u_{1}^{2}+u_{2}^{2}+u_{3}^{2}}}
$$

from $p_{H}$. If the sphere $\zeta(\mathbf{p})$ intersects the plane $p_{H}$ in a real circle, the intersection angle $\alpha$ is constant and given by

$$
\cos \alpha=\frac{d}{p_{4}}=\frac{u_{4}}{\sqrt{u_{1}^{2}+u_{2}^{2}+u_{3}^{2}}} .
$$

We see that this value only depends on $H$ and thus is the same for all image spheres. For further discussion we distinguish between the three cases from above:

(i) For a Euclidean hyperplane $\mathrm{H}$ we have $\cos ^{2} \alpha>1$ and thus the image spheres of all points in $H$ do not possess real intersection circles with the plane $p_{H}$ in $E^{3}$. However, admitting complex intersection angles $\alpha$, we see that this angle attains the same constant value for all spheres in $\zeta(H)$. The sphere set $\zeta(H)$ is either the set of points (spheres with radius zero) or it arises from that via an L-transformation.

(ii) If $H$ is a pseudo-Euclidean hyperplane, we get $\cos ^{2} \alpha<1$ and thus each sphere in the cyclographic image $\zeta(H)$ intersects the plane $p_{H}$ under the same constant angle $\alpha$. Via an $L$-transformation, we may achieve that this angle is a right one, i.e., that all spheres are centered in the plane $p_{H}$.

(iii) Under the cyclographic mapping, all points of an isotropic hyperplane $H$ get mapped to or. spheres tangent to the oriented plane $p_{H}$. This follows from $\cos ^{2} \alpha=1 \Longleftrightarrow u_{1}^{2}+u_{2}^{2}+u_{3}^{2}-u_{4}^{2}=0$.

Spheres in $\mathbb{R}^{3,1}$. A Minkowski 3-sphere $\Sigma \subset \mathbb{R}^{3,1}$ is an iso-distance set of a point $\mathbf{m}$ and therefore a quadric with an equation of the form

$$
\langle\mathbf{x}-\mathbf{m}, \mathbf{x}-\mathbf{m}\rangle=C=\text { const } \neq 0 .
$$

There are two cases: We may measure distances on space-like lines only, then $C=R^{2}$ is positive. To measure distances on time-like lines we have to take a negative constant value $C=-R^{2}$. 
The case $C=0$ represents a quadratic cone $\Delta$ (light cone). Such a cone intersects $E^{3}: x_{4}=0$ in a Euclidean sphere with center $\left(m_{1}, m_{2}, m_{3}\right)$ and radius $m_{4}$. Orienting this sphere $S$ with help of the sign of $m_{4}$ we see immediately that the cyclographic image spheres of the points of $\Delta$ are tangent to $S$.

We will mainly need 2-spheres, i.e., the hyperplanar intersections of 3spheres. It is sufficient to use a 3-sphere centered at the origin, $\Sigma:\langle\mathbf{x}, \mathbf{x}\rangle=C$, and study its intersection $S^{2}$ with the hyperplane

$$
H:\langle\mathbf{u}, \mathbf{x}\rangle+u_{0}=0
$$

We are interested in the cyclographic image spheres of the points of $S^{2}$. Those will be easy to characterize if we can show that there are light cones $\Delta$ passing through $S^{2}$. Let $\Delta$ have vertex $\mathbf{s}$. Then its equation reads $\Delta:\langle\mathbf{x}-\mathbf{s}, \mathbf{x}-\mathbf{s}\rangle=0$. The intersection $\Delta \cap \Sigma$ lies in a hyperplane $G$,

$$
G: 2\langle\mathbf{s}, \mathbf{x}\rangle-C-\langle\mathbf{s}, \mathbf{s}\rangle=0 .
$$

We see that we can choose s such that $G$ and $H$ agree: Comparing coefficients in their equations we obtain

$$
\mathbf{u}=2 \lambda \mathbf{s}, u_{0}=-\lambda(C+\langle\mathbf{s}, \mathbf{s}\rangle)
$$

Inserting $\mathbf{s}=\mathbf{u} /(2 \lambda)$ into the right hand equation, we find

$$
\lambda_{1,2}=\frac{1}{2 C}\left(-u_{0} \pm \sqrt{u_{0}^{2}-C\langle\mathbf{u}, \mathbf{u}\rangle}\right),
$$

and finally the vertex $\mathbf{s}=\mathbf{u} /(2 \lambda)$ of the light cone $\Delta$.

There are several cases to be discussed:

(i) $S^{2}$ is a Euclidean 2-sphere, in affine space $\mathbb{R}^{4}$ it is an ellipsoid. Here, the hyperplane $H$ is Euclidean, i.e. $\langle\mathbf{u}, \mathbf{u}\rangle<0$, and we can assume $\Sigma$ with $C>0$. Because of $D:=u_{0}^{2}-C\langle\mathbf{u}, \mathbf{u}\rangle>0$ we have two light cones passing through $S^{2}$. The vector connecting their vertices $\mathbf{s}_{1}, \mathbf{s}_{2}$ is parallel to $\mathbf{u}$ and thus it is light-like. This implies that the cyclographic image spheres $\zeta\left(\mathbf{s}_{i}\right)$ of the cone vertices do not have a common tangent cone (one lies inside the other or they agree up to their orientation). Thus, the cyclographic image of all points in $S^{2}$ consists of all oriented spheres which are tangent to $\zeta\left(\mathbf{s}_{1}\right)$ and $\zeta\left(\mathbf{s}_{2}\right)$.

(iia) $S^{2}$ is a pseudo-Euclidean 2-sphere with one sheet, in affine $\mathbb{R}^{4}$ a hyperboloid of one sheet. Now, $H$ is pseudo-Euclidean, i.e. $\langle\mathbf{u}, \mathbf{u}\rangle>0$, and we have to assume $\Sigma$ with $C>0$. In order to make sure that $S^{2}$ has only one sheet, $H$ must lie between those two tangent hyperplanes of $\Sigma$ which are parallel to $H$. It turns out that this is equivalent to $D<0$, implying that there is no real light cone passing through $S^{2}$.

(iib) $S^{2}$ is a pseudo-Euclidean 2-sphere with two sheets, in affine $\mathbb{R}^{4}$ a hyperboloid with two sheets. Again, $H$ is pseudo-Euclidean, i.e. $\langle\mathbf{u}, \mathbf{u}\rangle>0$, and we may assume $\Sigma$ with $C>0$, but now we have $D>0$. Hence, there are two real light cones passing through $S^{2}$. Their vertices $\mathbf{s}_{1}, \mathbf{s}_{2}$ are connected by 
a space-like line (parallel to $\mathbf{u}$ ) and thus the cyclographic image spheres $\zeta\left(\mathbf{s}_{i}\right)$ of the cone vertices possess a common tangent cone. Clearly, the cyclographic image of $S^{2}$ consists of those oriented spheres which are tangent to $\zeta\left(\mathbf{s}_{1}\right)$ and $\zeta\left(\mathbf{s}_{2}\right)$.

(iii) $S^{2}$ is an isotropic 2-sphere, in affine $\mathbb{R}^{4}$ it is an elliptic paraboloid. Here, $H$ is isotropic, $\langle\mathbf{u}, \mathbf{u}\rangle=0$, with $u_{0} \neq 0$. Hence, we obtain $\lambda_{1}=0$ and $\lambda_{2}=$ $-u_{0} / C$. Only $\lambda_{2}$ yields a valid cone vertex $\mathbf{s}_{2}$ with a cyclographic image sphere $\zeta\left(\mathbf{s}_{2}\right)$. The cyclographic image of $S^{2}$ consists of all spheres which are tangent to the oriented plane $p_{H}$ (see the discussion of isotropic hyperplanes above) and to the or. sphere $\zeta\left(\mathbf{s}_{2}\right)$.

\section{Edge offset meshes and their Laguerre invariance}

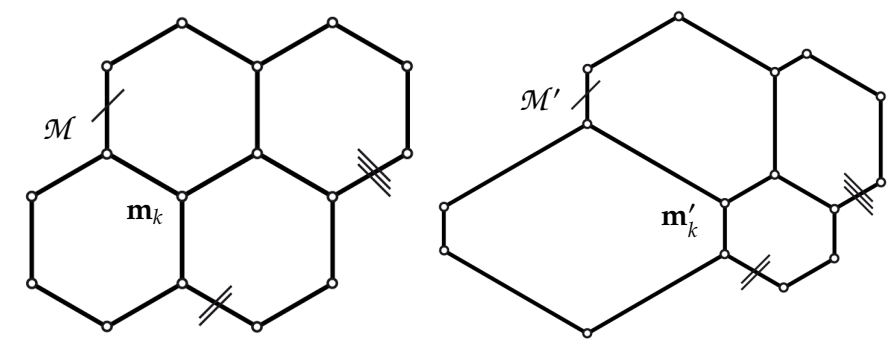

Fig. 2 Meshes $\mathcal{M}, \mathcal{M}^{\prime}$ with planar faces are parallel if they are combinatorially equivalent and corresponding edges are parallel.

Parallel meshes. A mesh $\mathcal{M}$ is represented by its vertices, concatenated in $\left(\mathbf{m}_{1}, \ldots, \mathbf{m}_{N}\right) \in \mathbb{R}^{3 N}$ and the combinatorics, i.e., edges and faces. If $\mathcal{M}^{\prime}, \mathcal{M}^{\prime \prime}$ have the same combinatorics, a linear combination $\lambda^{\prime} \mathcal{M}^{\prime}+\lambda^{\prime \prime} \mathcal{M}^{\prime \prime}$ is defined vertex-wise; this operation corresponds to the linear combination of vectors in $\mathbb{R}^{3 N}$. Meshes $\mathcal{M}, \mathcal{M}^{\prime}$ are parallel, if they have the same combinatorics and corresponding edges are parallel (see Fig. 2). We use this definition only if the faces of $\mathcal{M}$ (and hence of $\mathcal{M}^{\prime}$ ) are planar. Clearly, corresponding faces of $\mathcal{M}$ and $\mathcal{M}^{\prime}$ lie in parallel planes. The set of meshes parallel to $\mathcal{M}$ is denoted by $\mathcal{P}(\mathcal{M})$. The space $\mathcal{P}(\mathcal{M})$ is not rich for a triangle mesh $\mathcal{M}$ : Since triangles with parallel edges are scaled copies of each other, two parallel triangle meshes are scaled copies of each other. However, for other types of meshes with planar faces, mesh parallelism turned out to be essential for offset properties and an optimized layout of supporting beams in architectural designs based on such meshes (see [PLW*07] and Fig. 3).

Meshes with edge offsets. A mesh $\mathcal{M}^{\prime} \in \mathcal{P}(\mathcal{M})$ at constant distance from $\mathcal{M}$ is called an offset of $\mathcal{M}$. There are different ways to define the precise meaning of a constant offset distance, $\operatorname{dist}\left(\mathcal{M}^{\prime}, \mathcal{M}^{\prime}\right)=d$. We are interested here only in the case of edge offsets, where the distance of corresponding parallel edges (actually, lines which carry those edges) does not depend on 

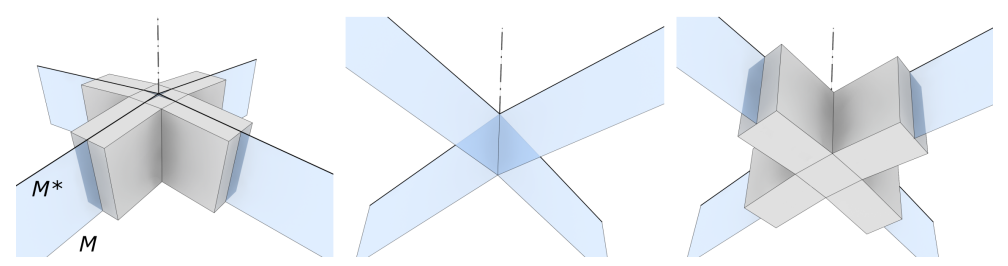

Fig. 3 Edge offset meshes are attractive candidates for architectural design since they give rise to the cleanest possible nodes in a supporting structure with beams of constant height.

the edge and equals $d$. For a pair $\mathcal{M}, \mathscr{M}^{\prime}$ of offset meshes one defines the socalled Gauss image mesh $\mathcal{S}=\left(\mathcal{M}^{\prime}-\mathcal{M}\right) / d$, which satisfies $\operatorname{dist}(\mathcal{S}, \mathbf{o})=1$ and is parallel to $\mathcal{M}$ and $\mathcal{M}^{\prime}$. Then, the following is easy to see [PLW*07]: $\mathcal{M}, \mathcal{M}^{\prime}$ is an edge offset pair if all edges of $\mathcal{S}$ are tangent to the Euclidean unit sphere $S^{2}$. Closed polyhedra $\mathcal{S}$ of that kind are known as Koebe polyhedra [Zie95]. A Koebe polyhedron is uniquely determined by its combinatorics up to a Möbius transformation (i.e., a projective mapping which transforms $S^{2}$ into itself); the computation from given combinatorics amounts to the minimization of a convex function [BS04]. Of course, in our case $\mathcal{S}$ needs not be closed and thus we speak of a Koebe mesh. For open Koebe meshes there is an additional degree of freedom for each boundary vertex.

If we call a mesh which possesses edge offsets an edge offset mesh (EO mesh), we can state the following fundamental result: $A$ mesh $\mathcal{M}$ is an edge offset mesh if and only if it is parallel to a Koebe mesh [PLW*07].

Let us mention a few well known and easily derived properties of Koebe meshes (Fig. 4): Each plane of a face $F_{k}$ of $S$ intersects $S^{2}$ in a circle $c_{F_{k}}$ which is tangent to the edges of that face; these circles form a packing. Per edge $e$ there is only one point of tangency $\mathbf{t}_{e}$ with the inscribed circles of the adjacent faces.
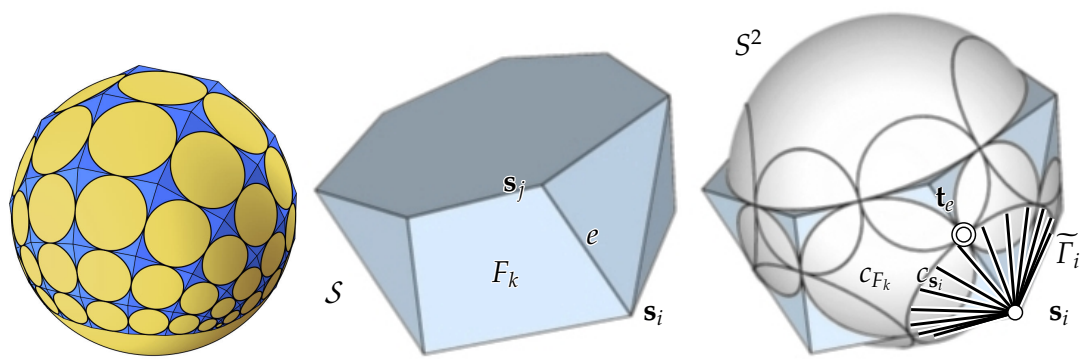

Fig. 4 Left: Inscribed circles of the faces in a Koebe mesh form a spherical circle packing (left). The edges emanating from a vertex of a Koebe mesh lie in a right circular cone which touches the sphere $S^{2}$ along a circle (right). These circles associated with the vertices form another circle packing which is orthogonal to the one defined by the faces.

Vertex cones. Since all edges emanating from a vertex $\mathbf{s}_{i}$ are tangents of $S^{2}$, they lie in a right circular cone $\widetilde{\Gamma}_{i}$ which touches $S^{2}$ along a circle $c_{\mathbf{s}_{i}}$ 
(Fig. 4). The circles associated with the vertices form another circle packing which is orthogonal to the packing formed by the in-circles of faces. Such orthogonal circle patterns have been first discussed by [Sch97]. The circle packing defined by the vertices defines the faces of another Koebe mesh $S^{*}$ which is the image of $S$ under the polarity with respect to $S^{2}$.

An edge offset mesh $\mathcal{M}$ is parallel to a Koebe mesh $\mathcal{S}$. Thus, also $\mathcal{M}$ has a vertex cone $\Gamma_{i}$ attached to each vertex $\mathbf{m}_{i} . \Gamma_{i}$ contains all edges through $\mathbf{m}_{i}$ and is parallel to the corresponding cone $\widetilde{\Gamma}_{i}$ at vertex $\mathbf{s}_{i} \in \mathcal{S}$. For an edge offset mesh $\mathcal{M}^{\prime}=\mathcal{M}+d \mathcal{S}$ of $\mathcal{M}$, the connecting line of corresponding vertices $\mathbf{m}_{i}, \mathbf{m}_{i}^{\prime}$ agrees with the common axis of the cones $\Gamma_{i}$ and $\Gamma_{i}^{\prime}$. Also by parallelity to $\mathcal{S}$ we see that the cones $\Gamma_{i}$ and $\Gamma_{j}$ to adjacent vertices $\mathbf{m}_{i}, \mathbf{m}_{j}$ of $\mathcal{M}$ are tangent to each other along the edge $\mathbf{m}_{i} \mathbf{m}_{j}$.

Laguerre invariance. An oriented right circular cone $\Gamma$ can be obtained as set of common or. tangent planes of two or. spheres. This shows that or. cones are invariant under Laguerre transformations if we include the limit cases of an or. cylinder of revolution and a straight line (cylinder of radius zero). In the following, we view an EO mesh as collection of oriented vertex cones; the orientation can be taken from an orientation of vertex cones in $\mathcal{S}$ which is derived from an oriented unit sphere $S^{2}$. We are now going to prove the following result, which has already been stated without proof in [PLW*07]:

Theorem 1 A Laguerre transformation $\kappa$ maps an edge offset mesh $\mathcal{M}$ to another edge offset mesh $\mathscr{M}^{\prime}=\kappa(\mathcal{M})$, if both are seen as the respective collection of vertex cones $\Gamma_{i}, \Gamma_{i}^{\prime}=\kappa\left(\Gamma_{i}\right)$.

Proof An L-transformation $\kappa$ maps or. vertex cones $\Gamma_{i}$ to or. cones $\Gamma_{i}^{\prime}$ and it keeps the tangency between adjacent cones. The vertex cones $\Gamma_{i}$ of $\mathcal{M}$ are parallel to the vertex cones $\widetilde{\Gamma}_{i}$ of the Koebe mesh $\mathcal{S}$; the latter cones are transformed by a Möbius transformation (projective automorphism of $S^{2}$ ) and thus they define the set of vertex cones $\widetilde{\Gamma_{i}^{\prime}}$ of a Koebe mesh $\mathcal{S}^{\prime}$ whose edges are tangent to $S^{2}$. Corresponding cones $\Gamma_{i}^{\prime}$ and $\widetilde{\Gamma_{i}^{\prime}}$ are parallel and thus even congruent. Moreover, adjacent cones $\Gamma_{i}^{\prime}$ and $\Gamma_{j}^{\prime}$ are tangent to each other along a common ruling (connecting line of their vertices $\mathbf{m}_{i}^{\prime}, \mathbf{m}_{j}^{\prime}$ ). Due to the parallel common tangent planes, this ruling $\mathbf{m}_{i}^{\prime} \mathbf{m}_{j}^{\prime}$ is parallel to the common ruling $\mathbf{s}_{i}^{\prime} \mathbf{s}_{j}^{\prime}$ of the parallel cones $\widetilde{\Gamma_{i}^{\prime}}$ and $\widetilde{\Gamma_{j}^{\prime}}$. This means that the vertices $\mathbf{m}_{i}^{\prime}$ of the cones $\Gamma_{i}^{\prime}$ form a mesh $\mathcal{M}^{\prime}$ which is parallel to the Koebe mesh $\mathcal{S}^{\prime}$ and therefore $\mathcal{M}^{\prime}$ is an EO mesh.

Note that the mapping from $\mathcal{M}$ to one of its edge offsets $\mathcal{M}^{\prime}=\mathcal{M}+d \mathcal{S}$ is also a Laguerre transformation and so the invariance of the class of EO meshes under edge offsetting appears now as a special case of Theorem 1.

Example 2 Here we show a pentagonal EO mesh, its Koebe mesh (Fig. 5) and a Laguerre transform (plus corresponding Koebe mesh).

Remark 3 It has been shown by [PL07] that EO meshes appear in the so-called isotropic model of Laguerre geometry as planar quad meshes, all whose faces have 
an inscribed isotropic circle with only one point of tangency per edge. These $i-$ isothermic meshes are an isotropic counterpart of a discretization of isothermic surfaces in Euclidean geometry according to [BHSO6] and are obviously invariant under isotropic Möbius transformations. Since that latter correspond to Laguerre transformations, we have another proof of Theorem 1.
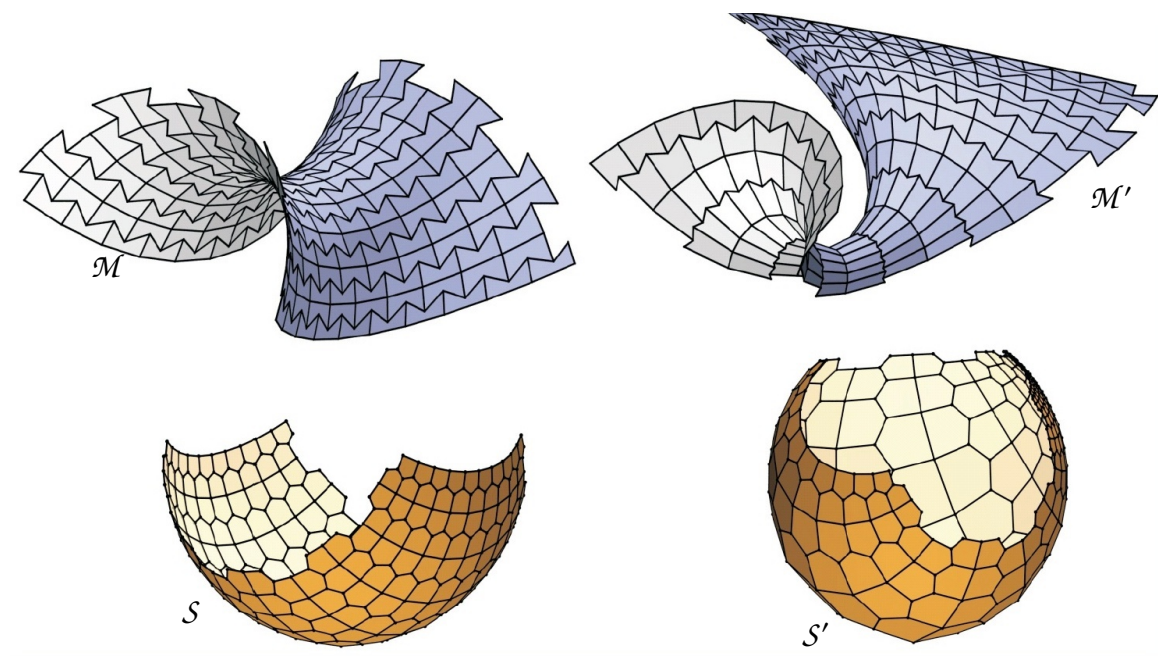

Fig. 5 The pentagonal EO mesh $\mathcal{M}^{\prime}$ (top right) arises from the EO mesh $\mathcal{M}$ (top left) by a Laguerre transformation. The corresponding parallel Koebe meshes (Gaussian images) $\mathcal{S}^{\prime}, \mathcal{S}$ are related by a Möbius transformation.

\section{The cyclographic image of EO meshes in hyperplanes of $\mathbb{R}^{3,1}$}

EO meshes in Euclidean, isotropic and pseudo-Euclidean 3-space. As we have seen earlier, the geometry in a hyperplane $H \subset \mathbb{R}^{3,1}$ is either Euclidean (e), pseudo-Euclidean (pe) or isotropic (i). In each type of space we can define $\mathrm{EO}$ meshes. We will show that the cyclographic image of an $E O$ mesh in a hyperplane of any type always yields a Euclidean EO mesh.

A Koebe mesh $\mathcal{S}$ in Euclidean 3-space $E^{3}$ is a polyhedral surface whose edges are tangent to an e-sphere $S_{e}^{2} \subset E^{3}$. An EO mesh $\mathcal{M} \subset E^{3}$ is parallel to a Koebe mesh $\mathcal{S}$. In a completely analogous way we define Koebe meshes and EO meshes in pseudo-Euclidean 3-space $P E^{3}$ and isotropic 3-space $I^{3}$. Of course, we have to use a sphere in that space. In pseudo-Euclidean 3-space with inner product $x_{1} y_{1}+x_{2} y_{2}-x_{3} y_{3}$, we are using a pe-sphere $S_{p e}^{2}$ with two sheets; it may be represented as $x_{1}^{2}+x_{2}^{2}-x_{3}^{2}=-1$. In isotropic 3-space $I^{3}$ based on the degenerate inner product $x_{1} y_{1}+x_{2} y_{2}$, the sphere is given by $2 x_{3}=$ $x_{1}^{2}+x_{2}^{2}$. From an affine perspective, the e-sphere is an ellipsoid, the pe-sphere is a hyperboloid with two sheets and the i-sphere is an elliptic paraboloid. 

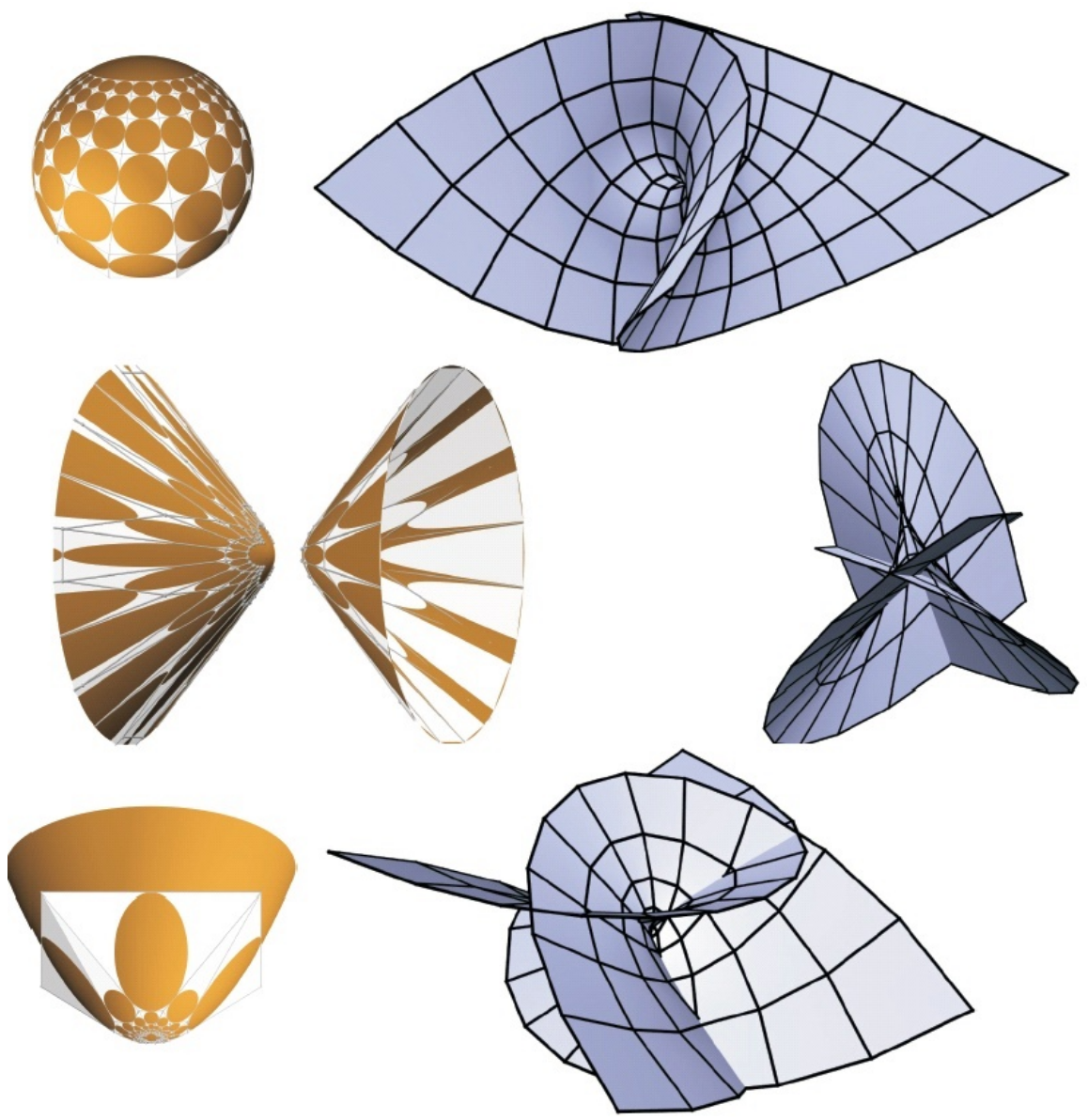

Fig. 6 Projectively equivalent Koebe meshes in Euclidean (top left), pseudo-Euclidean (middle left) and isotropic (bottom left) geometry. On the right, we see corresponding EO meshes (the Christoffel-dual discrete minimal surfaces).

However, from a projective perspective, these spheres are equivalent oval quadrics. Also the resulting e-, pe-, or i-Koebe meshes $S$ are projectively equivalent and thus can be easily computed with known methods. Of course, the projective equivalence does not extend to the derived parallel EO meshes. Fig. 6 shows three projectively equivalent Koebe meshes and associated parallel e-, pe- and i-EO meshes. These parallel meshes are even Christoffel-dual (see below) and thus represent discrete e-, pe- or i-minimal surfaces.

The properties we have mentioned above for e-Koebe and EO meshes hold in an analogous way for the pe- and i-counterparts. Most important are the vertex cones. These are right circular cones (within the corresponding geometry), namely tangent cones of spheres. 
The cyclographic image of a right circular cone. As a preparation for the proof of our next result on EO meshes, we investigate the cyclographic images of right circular cones:

Lemma 4 The cyclographic image of a right circular cone $\Gamma$ in an e- or pehyperplane $H \subset \mathbb{R}^{3,1}$ is a pair of oriented right circular cones $\zeta(\Gamma)_{1}, \zeta(\Gamma)_{2} \subset E^{3}$. If $H$ is isotropic, one of the two cones $\zeta(\Gamma)_{i}$ degenerates into an oriented plane.

Proof The cone $\Gamma$ may be defined as the envelope of a linear family of 2-spheres $S(t)$ defined by the vertex point $\mathbf{v}=S(0)$ and one inscribed 2-sphere, $S=S(1)$. Scaling $S(1)$ from center $\mathbf{v}$ with factor $t$ yields $S(t)$. As shown in section 2, in the e- and pe-case there are two light cones with vertices $\mathbf{s}_{1}(t), \mathbf{s}_{2}(t)$ passing through $S(t)$. Of course, we have $\mathbf{s}_{1}(0)=$ $\mathbf{s}_{2}(0)=\mathbf{v}$ and since scaling maps light cones to light cones, the set of vertices $\mathbf{s}_{1}(t)$ is a straight line $L_{1}$ through $\mathbf{v}$ and likewise $\mathbf{s}_{2}(t)$ is a line $L_{2}$ through $\mathbf{v}$. The cyclographic images of the points in $L_{1}$ are also cyclographic images of the spheres $S(t)$ and obviously they form a set of oriented spheres which envelope a right circular cone $\zeta(\Gamma)_{1}$; hence, this cone is part of the cyclographic image of $\Gamma$. Analogously, we obtain as image of $L_{2}$ the or. cone $\zeta(\Gamma)_{2}$ as the second component of the cylographic image of $\Gamma$. Note that the vertex of the cone $\zeta(\Gamma)_{i}$ is found by projecting $\mathbf{v}$ from the center $\mathbf{s}_{i}=\mathbf{s}_{i}(1)$ into $E^{3}$. In the i-case, we have only one center $\mathbf{s}_{1}$, but all image spheres of points in $H$ touch the oriented plane $H \cap E^{3}$ and thus the second part of the cyclographic image is this plane.

The cyclographic image of an EO mesh in a hyperplane of $\mathbb{R}^{3,1}$. The fact that right circular cones in hyperplanes are mapped to or. right circular cones in $E^{3}$ indicates that we can show the following theorem:

Theorem 5 The cyclographic image of an $E O$ mesh $\mathcal{M}$ in an e-or pe-hyperplane $H \subset \mathbb{R}^{3,1}$ is a pair of oriented Euclidean $E O$ meshes $\mathcal{M}_{1}, \mathcal{M}_{2}$ in $E^{3}$. In particular, a Koebe mesh $\mathcal{S} \subset H$ is mapped to a pair of projectively equivalent Euclidean Koebe meshes $\mathcal{S}_{1}, \mathcal{S}_{2} \subset E^{3}$. If $H$ is an $i$-hyperplane, the cyclographic image of an EO mesh (Koebe mesh) in $H$ consists of a Euclidean EO mesh (Koebe mesh, respectively) and a planar mesh.

Proof Let us first investigate the cyclographic image of the Koebe mesh $\mathcal{S} \subset M$ which is parallel to $\mathcal{M}$ and let us exclude the case of an isotropic hyperplane $H . S$ is tangent to a 2-sphere $S$, whose cyclographic image is a pair of or. spheres $S_{1}, S_{2} \subset E^{3}$. According to Lemma 4, the right circular cone $\Gamma_{i}$ associated with a vertex $\mathbf{v}_{i}$ of $\mathcal{S}$ gets mapped to a pair of or. cones $\zeta\left(\Gamma_{i}\right)_{1}, \zeta\left(\Gamma_{i}\right)_{2} \subset E^{3}$. The cone $\zeta\left(\Gamma_{i}\right)_{k}(k=1,2)$ is tangent to $S_{k}$ and its vertex $\mathbf{v}_{i}^{k}$ arises by projecting $\mathbf{v}_{i}$ from the vertex $\mathbf{s}_{k}$ of the light cone through $S$ into $E^{3}$. This means that the entire cone $\zeta\left(\Gamma_{i}\right)_{k}$ (as a point set) is obtained as image of $\Gamma_{i}$ under this central projection $\pi_{k}$. The projection only depends on $S$ and it maps $S$ onto $S_{k}$. Thus, $\pi_{k}$ maps the mesh $S$, which has planar faces and edges tangent to $S$, onto a mesh $\mathcal{S}_{k} \subset E^{3}$ whose faces are planar and whose edges are tangent to $S_{k}$. This shows that $\mathcal{S}_{k}$ is a Koebe mesh and that $\mathcal{S}_{1}$ and $\mathcal{S}_{2}$ are projectively equivalent. 
It should be noted that the inscribed sphere of a Koebe mesh $S_{k}$ may have vanishing radius; we would like to call this a Koebe mesh as well.

Since parallel cones in $H$ get mapped to pairs of parallel or. cones in $E^{3}$, the cyclographic image of an EO mesh $\mathcal{M} \subset H$ consists of two collections of cones each of which defines a mesh parallel to a Euclidean Koebe mesh $\left(\mathcal{S}_{1}\right.$ or $\left.\mathcal{S}_{2}\right)$ and hence the cyclographic image of $\mathcal{M}$ is a pair of oriented Euclidean EO meshes $\mathcal{M}_{1}, \mathcal{M}_{2}$.

The isotropic case is analogous and as in the proof of Lemma 4 we find that one component of the cyclographic image lies in the plane $H \cap E^{3}$.

If $H$ is a Euclidean hyperplane, we can move the EO mesh $\mathcal{M}$ via a Lorentz transformation into $E^{3}$ and get an EO mesh $\mathcal{M}^{\prime} \subset E^{3} . \mathcal{M}^{\prime}$ is not yet oriented and may be seen as two or. EO meshes $\mathcal{M M}_{1}^{\prime}, \mathcal{M}_{2}^{\prime}$. Since Lorentz transformations correspond to Laguerre transformations in the cyclographic image, the cyclographic image of $\mathcal{M}$ consists of the Laguerre transforms of the two or. EO meshes $\mathcal{M}_{1}^{\prime}, \mathcal{M}_{2}^{\prime}$. So we could apply Theorem 1 to prove Theorem 5 for the case of a Euclidean hyperplane $H$. However, since the latter theorem also includes the cases of EO meshes in i- and pe-hyperplanes $H$, it is more general than the L-invariance of EO meshes stated in Theorem 1.

Koebe pairs. A mesh pair $\left(\mathcal{S}_{1}, \mathcal{S}_{2}\right)$ arising as cyclographic image of a Koebe mesh $\mathcal{S}$ in a hyperplane $H \subset \mathbb{R}^{3,1}$ shall be called a Koebe pair. There are three types of Koebe pairs $\left(\mathcal{S}_{1}, \mathcal{S}_{2}\right)$. Depending on whether $H$ is Euclidean, pseudo-Euclidean or isotropic, we call the arising pair $\left(\mathcal{S}_{1}, S_{2}\right)$ an e-pair, pe-pair or $i$-pair, respectively.

Construction and properties from the Euclidean L-geometric perspective. We now turn to the question of how to construct a Koebe pair from a Koebe mesh $S_{1}$ tangent to a sphere $S_{1} \subset E^{3}$. It turns out that it is possible to prescribe a second sphere $S_{2} \subset E^{3}$ and to find a unique Koebe mesh $S_{2}$ tangent to $S_{2}$ such that $\left(\mathcal{S}_{1}, \mathcal{S}_{2}\right)$ is a Koebe pair (cyclographic image of a Koebe mesh $S$ ). Let $S^{2} \subset \mathbb{R}^{3,1}$ be the 2-sphere determined by $S_{1}, S_{2}$ with carrier hyperplane $H$. We first assume that there exists a Koebe mesh $\mathcal{S}$ tangent to $S^{2}$ with cyclographic image $\left(\mathcal{S}_{1}, \mathcal{S}_{2}\right)$. Take a vertex cone $\Gamma_{i}$ of $S$ tangent to $S^{2}$ and consider its intersection conic $c_{i}$ with $S^{2}$. Clearly, the cyclographic image of $c_{i}$ consists of all spheres which are tangent to $\zeta\left(\Gamma_{i}\right)_{1}, \zeta\left(\Gamma_{i}\right)_{2}, S_{1}$ and $S_{2}$. Their midpoints are given as the top view of $c_{i}$. This observation yields the following construction of $\mathcal{S}_{2}$, if $\mathcal{S}_{1}$ is given: For a vertex cone $\Gamma_{1}$ of $S_{1}$ find all spheres which are tangent to $\Gamma_{1}, S_{1}$ and $S_{2}$. These spheres touch $S_{2}$ in a curve. If $\mathcal{S}$ exists, this curve is a planar section of $S_{2}$, and we get the vertex of $\mathcal{S}_{2}$ corresponding to the vertex of $\Gamma_{1}$ as the polarity of this plane with respect to $S_{2}$. Note that this construction is invariant with respect to Möbius transformations (=incidence preserving transformations acting bijectively on spheres and points), if the Koebe meshes $\mathcal{S}_{1}$ and $\mathcal{S}_{2}$ are viewed as spherical circle packings (cf. Fig. 7 top right). At this point it is however not clear if it actually yields a Koebe pair. The following theorem shows that it does: 
Theorem 6 Given two (non-intersecting) spheres $S_{1}, S_{2} \subset E^{3}$ and a Koebe mesh $S_{1}$ tangent to $S_{1}$ there exists a unique Koebe pair $\left(S_{1}, S_{2}\right)$ where $S_{2}$ is tangent to $S_{2}$. The same holds if one of $S_{1}$ or $S_{2}$ is a plane.

Proof Let $S^{2} \subset H \subset \mathbb{R}^{3,1}$ be the 2-sphere determined by $S_{1}, S_{2}$ as intersection of the isotropic light cones emanating from $\zeta^{-1}\left(S_{1}\right)$, resp. $\zeta^{-1}\left(S_{2}\right)$. $H$ is the hyperplane carrying $S^{2}$. We show how to construct the preimage $\mathcal{S}$ of the Koebe pair. It is tangent to the 2-sphere $S^{2} \subset H \subset \mathbb{R}^{3,1}$ determined by $S_{1}, S_{2}$ as intersection of the isotropic light cones emanating from $\zeta^{-1}\left(S_{1}\right)$, resp. $\zeta^{-1}\left(S_{2}\right) . H$ is the hyperplane carrying $S^{2}$. Let $\Gamma_{1}$ be a cone tangent to $S_{1}$. The preimage $\zeta^{-1}\left(\Gamma_{1}\right)$ is given by a straight line $L_{1}$ through $S_{1}$. Let $\Delta_{1}(t)$ be the one parameter family of light cones emanating from points of $L_{1}$. The intersection of $\Delta_{1}(t)$ with $H$ determines a linear one parameter family of 2 -spheres including $S^{2}$. They envelope a cone $\Gamma$ tangent to $S^{2}$. Note that $\Gamma$ is the image of $\Gamma_{1}$ under a central projection $\pi_{1}^{-1}: E^{3} \rightarrow H$ depending only on $S^{2}$ (compare the proof of Theorem 5). This implies that we obtain a Koebe mesh $\mathcal{S}$ tangent to $S^{2}$ by transferring all vertex cones of $\mathcal{S}_{1}$ in the above described fashion. The case that $S_{2}$ is a plane is analogous. In that case $H$ is an isotropic hyperplane. If $S_{1}$ is a plane, we apply a Möbius transformation $\Phi$ to map $S_{1}$ into a sphere. This way we transfer the situation to the case already treated and obtain a Koebe pair $\left(\Phi\left(\mathcal{S}_{1}\right), \Phi\left(\mathcal{S}_{2}\right)\right)$ tangent to $\Phi\left(S_{1}\right)$ resp. $\Phi\left(S_{2}\right)$. Applying the inverse Möbius transformation $\Phi^{-1}$ yields two Koebe meshes $\mathcal{S}_{1}, \mathcal{S}_{2}$ tangent to $S_{1}, S_{2}$. We have now a pair of Koebe meshes $\mathcal{S}_{1}, S_{2}$ related by the Möbius invariant construction described above. It follows from the first part of the proof, that there exists a Koebe pair $\left(S_{1}^{\prime}, S_{2}\right)$ where $S_{1}^{\prime}$ is tangent to $S_{1}$. By our previous discussion $S_{1}^{\prime}$ is also related to $\mathcal{S}_{2}$ by the construction described above. From the uniqueness of this construction it follows that $S_{1}^{\prime}=S_{1}$, and hence $\left(\mathcal{S}_{1}, \mathcal{S}_{2}\right)$ is a Koebe pair. This concludes the proof.

The Möbius and Laguerre transformation groups naturally occur as subgroups of the more general group of Lie sphere transformations which consists of all incidence preserving transformations acting on the set of oriented spheres, points and oriented planes [Cec92]. Moreover, the Möbius and Laguerre groups generate the group of Lie sphere transformations. The previous theorem implies the Möbius invariance of Koebe pairs and being a Koebe pair is also clearly a Laguerre invariant property. We arrive at the following corollary:

Corollary 7 Being a Koebe pair is a Lie invariant property.

We translate some properties of a Koebe mesh $S$ in a hyperplane $H$ and tangent to $S^{2} \subset H$ to the respective properties of the associated Koebe pair $\left(S_{1}, S_{2}\right)$ tangent to $S_{1}, S_{2} \in E^{3}$, respectively:

A vertex $p_{i} \in \mathbb{R}^{4}$ of $\mathcal{S}$ corresponds to a sphere in Euclidean space. It can be constructed directly as the intersection of the two cones in $E^{3}$ obtained as cyclographic image of the cone in $H$ through $p_{i}$ of $S$ tangent to $S^{2}$. ( see Fig.8 ). We call these spheres vertex spheres. The two corresponding cones 

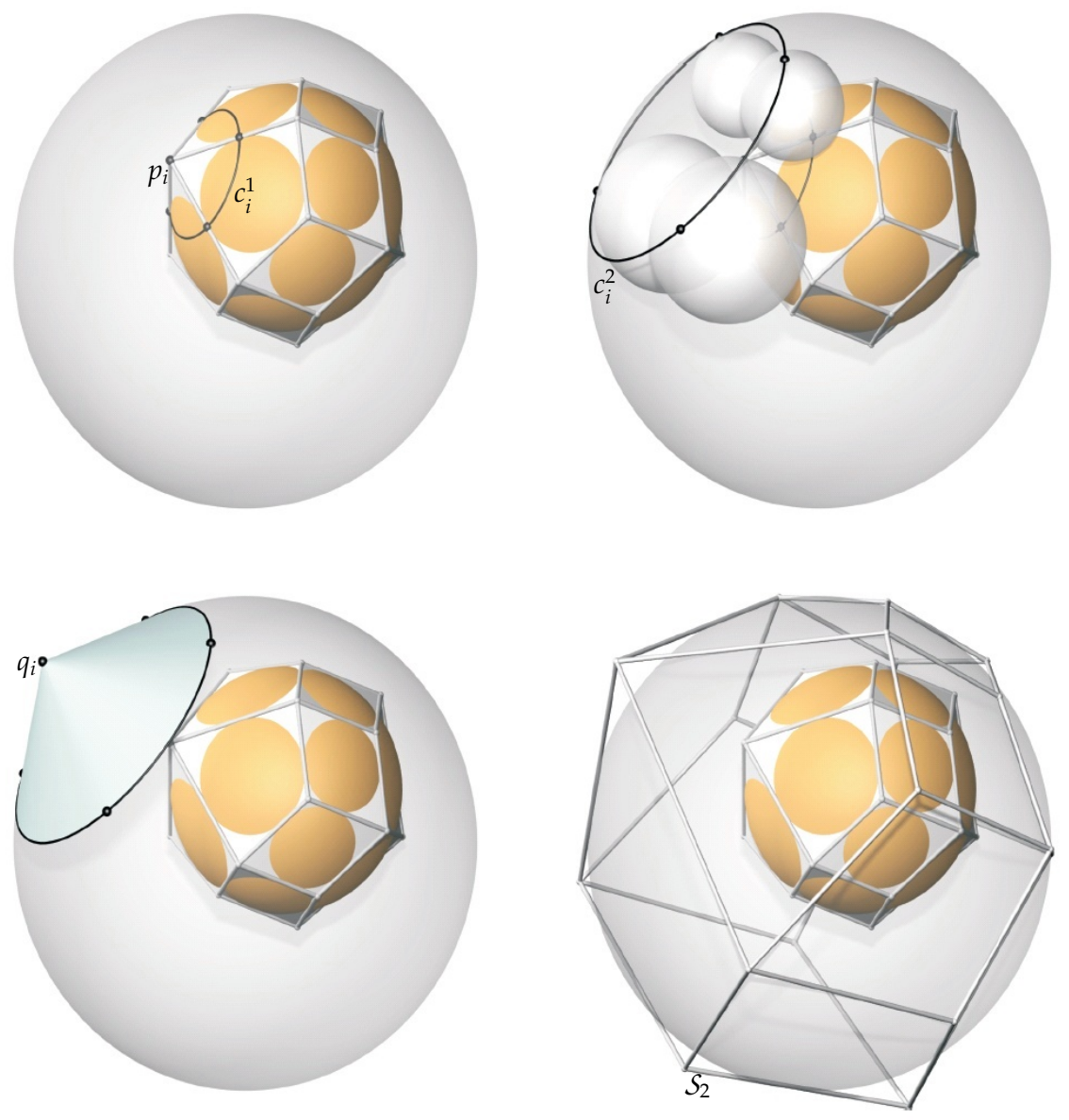

Fig. 7 Pointwise construction of a Koebe Pair given a Koebe mesh $\mathcal{S}_{1}$ tangent to a sphere $S_{1}$ and a second sphere $S_{2}$ (top left): First, the intersection curve $c_{i}^{1}$ of $S_{1}$ with the polar hyperplane of $p_{i}$ with respect to $S_{1}$ is computed (top left). Then all spheres are found which touch $S_{1}$ in $c_{i}$ and $S_{2}$. The intersection curve of all these spheres with $S_{2}$ is a circle $c_{i}^{2}$ on $S_{2}$ (top right, four such spheres are shown). Computing the polarity of the carrier plane of $c_{i}^{2}$ with respect to $S_{2}$ we arrive at the corresponding point $q_{i}$ which belongs to the second Koebe mesh $S_{2}$ tangent to $S_{2}$ (bottom left). If we repeat this construction for every point of $\mathcal{S}_{1}$, we arrive at a second Koebe mesh $\mathcal{S}_{2}$ tangent to $S_{2}$ such that $\left(\mathcal{S}_{1}, \mathcal{S}_{2}\right)$ is a Koebe pair (bottom right).

touching the vertex spheres are the vertex cones of the Koebe meshes $\mathcal{S}_{1}$ and $\mathcal{S}_{2}$.

The faces of the Koebe mesh $\mathcal{S}$ possess incircles, where the term "circle" here means a planar section of the sphere $S^{2}$ in $H$ to which the edges of $S$ are tangent. In the Euclidean setting this means that the edge cones each possess an inscribed cyclide. We call them face cyclides (cf. Fig. 8). 

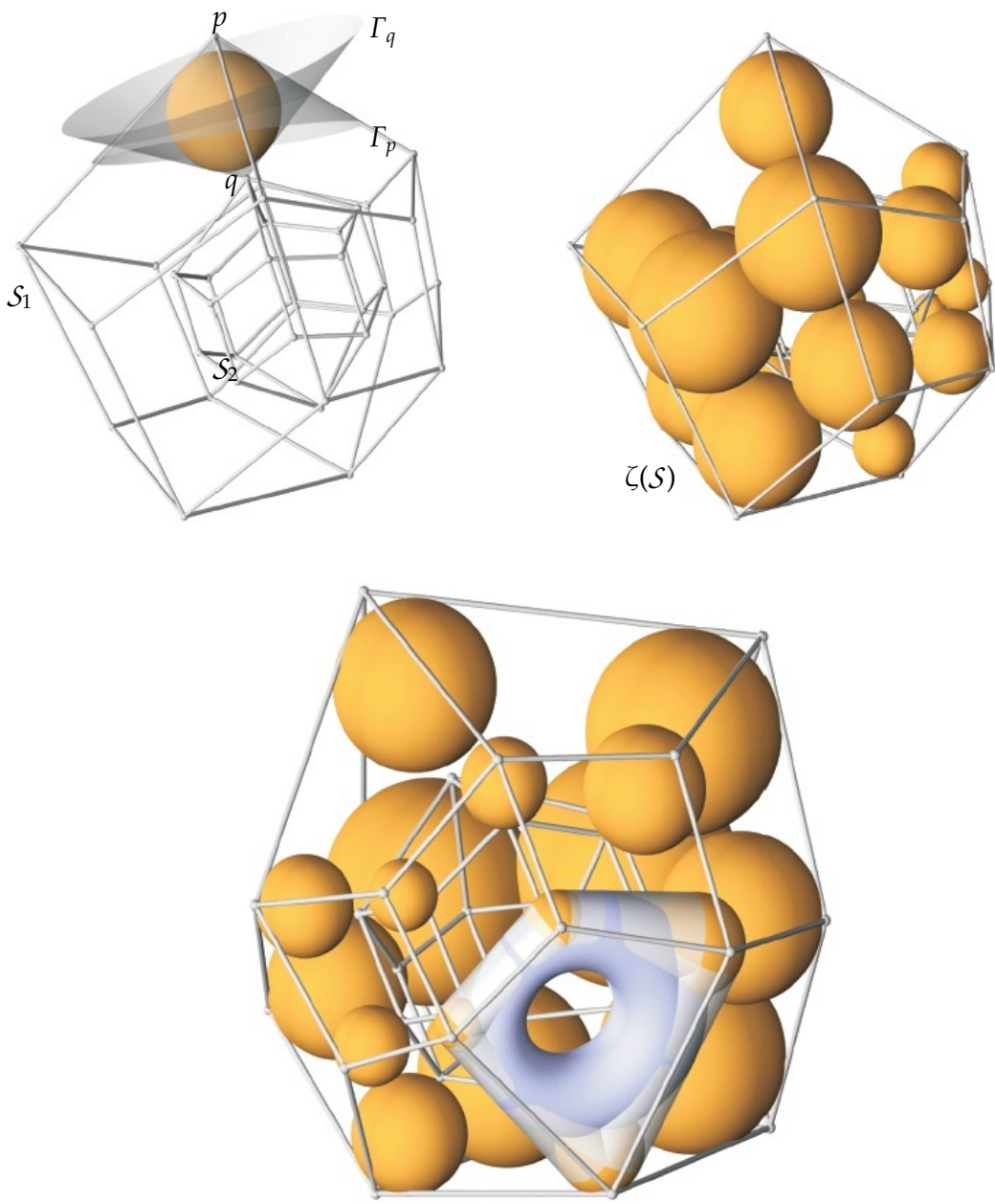

Fig. 8 Top: Construction of the middle spheres of a Koebe pair $\left(\mathcal{S}_{1}, \mathcal{S}_{2}\right)$ corresponding to a Koebe mesh $\mathcal{S}$ in a hyperplane $H \subset \mathbb{R}^{3,1}$ : For every pair of corresponding points $p, q$ of $\mathcal{S}_{1}, S_{2}$ we compute the intersection sphere of the vertex cones $\Gamma_{p}$ and $\Gamma_{q}$ through $p, q$ respectively. Bottom: Edge cones of every face possess an incyclide, the face-cyclide.

By considering the top-view $\pi(\mathcal{S})$ of $\mathcal{S}$, we see that the midpoints of the vertex spheres also form a Koebe mesh $S_{M}$, this time with respect to the quadric $S_{M}:=\pi(S)$. This may be interpreted as discrete version of an isothermic parametrization of $S_{M}$ with respect to the metric induced from the quadric $S_{M}$.

The construction of the Koebe mesh $\mathcal{S}_{2}$ from $\mathcal{S}_{1}$ admits an interpretation in terms of geometrical optics: $\mathcal{S}_{2}$ can be seen as a discrete anticaustic of $S_{M}$ for light-rays emanating from the midpoint $m_{1}$ of $S_{1}$. This is clear, since $S_{2}$ itself is an anticaustic of $S_{M}$ for light-rays emanating from $m_{1}$. If $S_{1}$ is a 


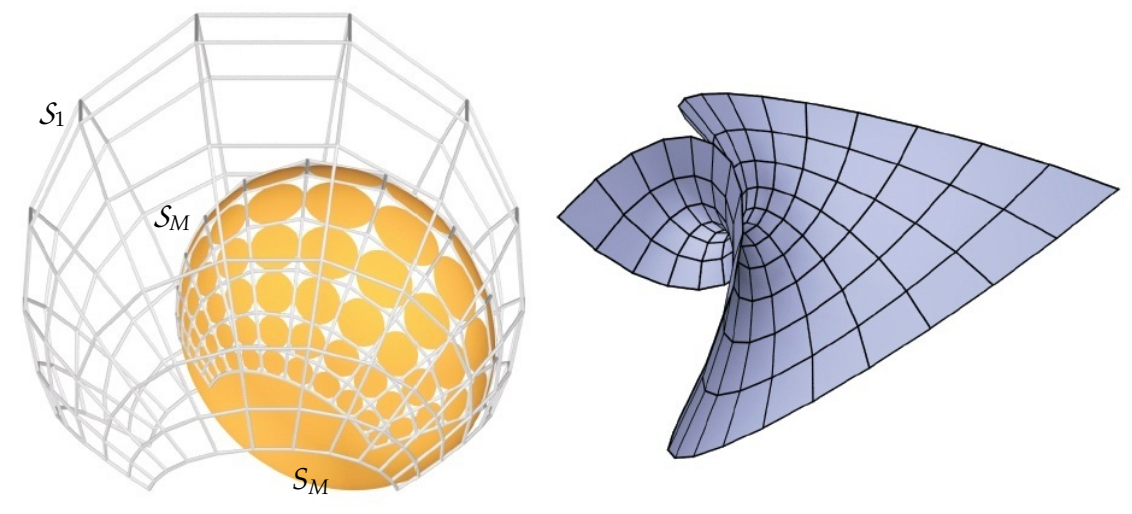

Fig. 9 Left: The top view $\mathcal{S}_{M}:=\pi(S)$ of a Koebe mesh $S$ tangent to a 2-sphere $S^{2}$ in a hyperplane $H \subset \mathbb{R}^{3,1}$ is a Koebe mesh tangent to the quadric $S_{M}:=\pi\left(S^{2}\right) \subset E^{3}$. Our figure depicts the Euclidean case. The second Koebe mesh $S_{2}$ of the Koebe pair $\left(\mathcal{S}_{1}, S_{2}\right)$ corresponding to $S$ lies on the inside of $S_{M}$ and is not shown in this figure. Right: The Christoffel dual of $S_{M}$ is a minimal surface with respect to the metric induced by $S_{M}$. It consists of the middle spheres of the L-minimal surface $\mathcal{M}$ which is Christoffel dual to $S$.

plane, this midpoint is defined to be the point at infinity of the direction orthogonal to $S_{1}$.

\section{Edge offset meshes which are discrete Laguerre minimal surfaces}

Laguerre minimal surfaces and Laguerre isothermic surfaces. An orientable surface $\Phi \subset E^{3}$ can be represented in Laguerre-invariant form as envelope of so-called contact elements $(p, T)$, where $p \in \Phi$ and $T$ is the oriented tangent plane of $\Phi$ in $p$. The contact element $(p, T)$ represents all oriented spheres touching the surface $\Phi$ in $p$. As such it is invariant under Laguerre transformations since the spheres of a contact element form an isotropic line in $\mathbb{R}^{3,1}$. One way to study the L-differential geometry of $\Phi$ is to pick one specific oriented sphere $M$ in every contact element of $\Phi$ in an invariant way, and to study the differential geometry of the twoparameter family of these spheres embedded in $\mathbb{R}^{3,1}$. The usual choice of $M$ is the midpoint of the cyclographic preimages $\zeta^{-1}\left(\Gamma_{1}\right), \zeta^{-1}\left(\Gamma_{2}\right)$ of the two principal spheres $\Gamma_{1}, \Gamma_{2}$ in $p$ with midpoints $m_{1}, m_{2}$ and radii $R_{1}, R_{2}$. This means that the midpoint of $M$ is given as $m=\frac{1}{2}\left(m_{1}+m_{2}\right)$ and the radius $R=\frac{1}{2}\left(R_{1}+R_{2}\right) . M$ is called the middle sphere of $\Phi$ at $p$. Laguerre differential geometry of $\Phi$ is the differential geometry of the 2-surface $\Phi_{M} \subset \mathbb{R}^{3,1}$ which is formed by all points $M$ corresponding to the middle spheres of $\Phi$.

It is worth noting that the mapping that takes $\Phi$ to $\Phi_{M}$ is a conformal mapping if we choose the metric induced by the third fundamental form on $\Phi$ [Pal99]. This indicates that the spherical image of a surface plays an 
important role in the L-differential geometry of $\Phi . \Phi_{M}$ is called the L-Gauss image.

A surface $\Phi$ is called Laguerre-isothermic if its spherical image yields an isothermic parametrization of the unit sphere. We immediately see that the notion of an EO-mesh is the right discrete analogue of an L-isothermic surface, since Koebe polyhedra correspond to isothermic parameterizations of the Euclidean unit sphere and we know that the spherical image of an EO-mesh is a Koebe polyhedron.

A Laguerre minimal surface is defined as a surface whose image $\Phi_{M} \subset \mathbb{R}^{3,1}$ is a local minimizer of the area functional $\Omega$ in $\mathbb{R}^{3,1}$. Applied to $\Phi$, it may be written as

$$
\Omega=\int\left(R_{1}-R_{2}\right)^{2} d A_{s},
$$

where $d A_{s}=K d A$ means the surface element of $\Phi_{M}$ and $R_{1}, R_{2}$ are the principal radii.

Not much seems to be known about the shape properties of general $L$ minimal surfaces, but if in addition the surface is required to be isothermic, there is the following classical result by [Bla29, Page 375]:

Theorem 8 An L-isothermic L-minimal surface $\Phi$ must belong to the following three types of surfaces (up to L-trafos):

(i) A Euclidean minimal surface,

(ii) an L-minimal surface of the spherical type where all middle spheres are tangent to a fixed plane,

(iii) a Bonnet surface where all midpoints of the middle spheres lie on a fixed plane.

In particular, $\Phi_{M}$ must lie in a Euclidean, isotropic, or pe-hyperplane $H$ of $\mathbb{R}^{3,1}$.

L-minimal EO meshes. We discuss the definition and construction of Lminimal EO-meshes. According to Theorem 8 their L-Gauss image must lie in a Hyperplane $H \subset \mathbb{R}^{3,1}$. Therefore we first treat the construction of discrete minimal surfaces of $\mathbb{R}^{3,1}$ in a hyperplane: Let us start with a hyperplane $H$ with inner product $\langle\cdot, \cdot\rangle_{H} \cdot H$ can either be Euclidean, isotropic or pseudo-Euclidean. Since any two hyperplanes of the same type can be mapped into each other by an L-trafo, all the metrics we need to consider are the ones induced by the following inner products in $\mathbb{R}^{3}$ :

(e) $\langle\mathbf{x}, \mathbf{y}\rangle_{e}=x_{1} y_{1}+x_{2} y_{2}+x_{3} y_{3}$

(i) $\langle\mathbf{x}, \mathbf{y}\rangle_{i}=x_{1} y_{1}+x_{2} y_{2}$,

(pe) $\langle\mathbf{x}, \mathbf{y}\rangle_{p e}=x_{1} y_{1}+x_{2} y_{2}-x_{3} y_{3}$.

In order to be able to speak about discrete minimal surfaces with respect to these metrics, we need to introduce discrete mean curvatures associated with the faces of an EO-mesh $\mathcal{M}$. This has been done for the cases $(e)$ and (i) in [PLW*07, PL07] and the case (pe) is similar. Here, we only discuss the pe-case: Let $F$ be an oriented face of a mesh $\mathcal{M}$ with vertices $v_{0}, \ldots, v_{k-1}$. Its spherical image $S=\sigma(F)$ with edges tangent to the pe-unit sphere $x_{1}^{2}+x_{2}^{2}-$ $x_{3}^{2}=-1$ shall have vertices $s_{0}, \ldots, s_{k-1}$. The existence of $S$ is guaranteed by 
the fact that $\mathcal{M}$ is an EO-mesh. The area of $F, S$ w.r.t. the metric induced by the inner product $(p e)$ is given by

$$
\operatorname{area}(F)_{p e}=\frac{1}{2} \sum_{j=0}^{k-1} \operatorname{det}\left(v_{j}, v_{j+1}, n\right), \operatorname{area}(S)_{p e}=\frac{1}{2} \sum_{j=0}^{k-1} \operatorname{det}\left(s_{j}, s_{j+1}, n\right),
$$

where indices are understood modulo $k$ and $n$ is the normal vector with respect to the inner product $\langle\cdot, \cdot\rangle_{p e}$ of the carrier plane of $F$ and $S$.

The pe-mixed area area $(F, S)_{p e}$ of two parallel polygons $F, S$ with normal vector $n$ is defined by

$$
\operatorname{area}(F, S)_{p e}:=\frac{1}{4} \sum_{j=0}^{k-1}\left[\operatorname{det}\left(v_{j}, s_{j+1}, n\right)+\operatorname{det}\left(s_{j}, v_{j+1}, n\right)\right] .
$$

The pe-mean curvature of the face $F$ is defined as

$$
H(F)_{p e}:=\frac{\operatorname{area}(F, S)_{p e}}{\operatorname{area}(F)_{p e}} .
$$

This definition shares many properties with its smooth counterpart. For more information we refer to [PLW*07]. We define an EO-mesh $\mathcal{M}$ to be minimal if for each face $F$ the pe-mean curvature $H(F)_{p e}$ vanishes, i.e. if

$$
\operatorname{area}(F, \sigma(F))_{p e}=0 \quad \text { for all faces } F \text { of } \mathcal{M} \text {. }
$$

Observe that the condition (8) does not depend on the choice of the normal vector $n$. If we used the usual Euclidean normal vector instead of $n$, the condition would not change. With this modification, the definition of mixed area area $(F, S)_{p e}$ coincides with the usual notion of mixed area area $(F, S)$ known from convex geometry [Zie95]. With similar reasoning one arrives at analogous definitions and results in the cases (e), (i) (cf. [PLW*07, PL07]). For quadrilateral parallel faces $F, S$ there exists a simple geometric criterion for them to have vanishing mixed area (cf. [PLW*07]):

Theorem 9 Two parallel quads $F$ and $S$ with vertices $v_{0}, v_{1}, v_{2}, v_{3}$ and $s_{0}, s_{1}, s_{2}, s_{3}$ have vanishing mixed area iff the diagonals of $F$ and $S$ are antiparallel, i.e. $v_{0} v_{2} \| s_{1} s_{3}$ and $v_{1} v_{3} \| s_{0} s_{2}$.

The general recipe for constructing a minimal $E O$-mesh $\mathcal{M}$ from its spherical image $S=\sigma(\mathcal{M})$ in a hyperplane $H \subset \mathbb{R}^{3,1}$ is to use the above theorem face-wise. The resulting mesh $\mathcal{M}$ is called Christoffel-dual of $\mathcal{S}$ and it is an EO-mesh in $H$. By Theorem 5 the cyclographic image of $\mathcal{M}$ consists of two Euclidean EO-meshes $\mathcal{M}_{1}, \mathcal{M}_{2}$. We call such meshes L-minimal EO-meshes. We have just proved the following theorem:

Theorem 10 Let $H \subset \mathbb{R}^{3,1}$ be a hyperplane and $S^{2}$ a 2-sphere in $H$. Then a discrete $L$-isothermic L-minimal surface realized as an EO mesh can be constructed as follows: 
1. construct a Koebe polyhedron $S$ tangent to $S^{2}$. This may be achieved by constructing a Koebe mesh tangent to the Euclidean unit sphere and mapping it to a Koebe mesh tangent to $S^{2}$ using an appropriate projective transformation (cf. Fig. 6).

2. apply the Christoffel duality to $\mathcal{S}$ to obtain a minimal surface $\mathcal{M} \subset H$ of $\mathbb{R}^{3,1}$.

3. The cyclographic image $\zeta(\mathcal{M})$ yields by Theorem 5 a pair $\left(\mathcal{M}_{1}, \mathcal{M}_{2}\right)$ of Euclidean EO meshes which are discrete models of L-isothermic L-minimal surfaces.

The spheres corresponding to the points of $\mathcal{M}$ are the discrete middle spheres of $\left(\mathcal{M}_{1}, \mathcal{M}_{2}\right)$.

Since any Euclidean hyperplane can be mapped into $x_{4}=0$, any isotropic hyperplane into $x_{1}-x_{4}=0$ and any pe-hyperplane into $x_{1}=0$ by a L-trafo, we arrive at the following theorem which is a discrete analogue of Theorem 8:

Theorem 11 Up to L-trafos, L-isothermic L-minimal EO meshes belong to the following three types, depending on $\mathrm{H}$ being Euclidean, isotropic, or pe:

(a) A discrete Euclidean minimal surface,

(b) a discrete L-minimal surface of the spherical type where all middle spheres are tangent to a fixed plane,

(c) a discrete Bonnet surface where all midpoints of the middle spheres lie on a fixed plane.

The midpoints of the middle spheres comprise a Koebe mesh $S_{M}$ in $E^{3}$ tangent to the top view $S_{M}=\pi\left(S^{2}\right)$ of $S^{2}$. The top view $\pi(\mathcal{M})$ of the Christoffel dual $\mathcal{M}$ of $\mathcal{S}$ in $H$ clearly is the Christoffel dual of $\mathcal{S}_{M}$ since pairs of parallel edges get mapped to pairs of parallel edges under the top view projection. Therefore $\pi(\mathcal{M})$ is a minimal surface with respect to the metric induced by the quadric $S_{M}$ (cf. Fig. 9). Special attention has to be paid to the case that $H$ is parallel to the vector $(0,0,0,1)$ which happens only if $H$ is a pe-plane. In this case $S_{M}$ is not a quadric, but a plane.

Since $\pi(\mathcal{M})$ consists of the midpoints of the middle spheres of $\mathcal{M}$, we arrive at the following theorem:

Theorem 12 If $H$ is a hyperplane in $\mathbb{R}^{3,1}$ not parallel to the vector $(0,0,0,1)$, and $\mathcal{M}$ is a discrete $L$-minimal surface in $H$, then the midpoints of $\mathcal{M}$ comprise a minimal surface with respect to the metric induced by $S_{M}$. If $(0,0,0,1)$ is parallel to $H$, then the midpoints of the middle spheres of $\mathcal{M}$ are the image of a pe-minimal surface under a parallel projection.

\section{Euclidean construction of L-minimal surfaces and examples}

For computational purposes, among other reasons, it is of course desirable to have a construction of L-minimal EO meshes at hand that avoids the detour in Minkowski space and that operates simply in $E^{3}$. To achieve this, we need to translate the Christoffel duality construction in a hyperplane into a construction in $E^{3}$. Using Theorem 9 this is easy since parallel edges in $H$ get mapped into parallel cones in $E^{3}$. We therefore define the L-Christoffel 
duality in $E^{3}$ to be the same as the usual Christoffel duality, with edges replaced by cones, and parallelity of edges by parallelity of cones. The goal is to construct a (pair of) L-minimal EO mesh(es) given a Koebe pair $\left(\mathcal{S}_{1}, \mathcal{S}_{2}\right)$. The Koebe pair may for example be constructed according to Theorem 6 . Let us assume that $\left(\mathcal{S}_{1}, \mathcal{S}_{2}\right)$ corresponds to a Koebe mesh $S$ in a hyperplane $H \subset \mathbb{R}^{3,1}$ with Christoffel dual $\mathscr{M} \subset H$. The cyclographic image $\zeta(S)$ of the points of $\mathcal{S}$ is given by the vertex spheres of $\left(S_{1}, S_{2}\right)$. They are constructed as the intersection sphere of corresponding vertex cones of $S_{1}$ and $S_{2}$ viewed as linear one-parameter families of spheres (see Figure 8). Now we apply the L-Christoffel duality to the mesh $\zeta(S)$ face-wise according to Figure 10 to obtain the L-Christoffel-dual sphere-valued mesh $\zeta(\mathcal{M})$. This mesh consists of the middle spheres of the L-minimal surface. It remains to compute the envelope of $\zeta(\mathcal{M})$ which, according to Theorem 5, consists of a pair of EOmeshes $\mathcal{M}_{1}, \mathcal{M}_{2}$ with $\mathcal{M}_{i}$ parallel to $\mathcal{S}_{i}, i=1,2$. Because of this parallelity, the vertex cones of $\mathcal{M}_{i}$ must be parallel to the vertex cones of $\mathcal{S}_{i}$. Consequently, to construct the points of $\mathcal{M}_{i}$ it suffices to parallel translate the vertex cones of $S_{i}$ into corresponding spheres of $\zeta(\mathscr{M})$. The points of these translated cones are the points of $\mathcal{M}_{i}$. There are two further simplifications that we can take advantage of: First note that the midpoints of $\zeta(\mathcal{M})$ are given as the usual Christoffel transform of the midpoints of $\zeta(\mathcal{S})$. This simplifies the L-Christoffel duality construction. Second, it is no loss of generality if we restrict ourselves to only three hyperplanes $H \subset \mathbb{R}^{3,1}$ : one Euclidean hyperplane $H_{e}$, one isotropic hyperplane $H_{i}$ and one pe-hyperplane $H_{p e}$. This is because two e-, i-, or pe-hyperplanes can be mapped into each other by an L-trafo. Recall that a quadric in a hyperplane $H$ is always determined by the two spheres $S_{1}, S_{2}$ with the property that every sphere in the cyclographic image of the quadric touches $S_{1}$ and $S_{2}$.

The Euclidean case: There is not much to say in this case. We can let the spheres $S_{1}, S_{2}$ agree up to their orientation. A Koebe mesh $\mathcal{S}$ tangent to $S_{1}$ (and $S_{2}$ ) already represents a Koebe pair. The middle spheres of this Koebe pair are given by the points of $\mathcal{S}$, and therefore the L-Christoffel duality of the middle spheres coincides with the conventional Christoffel duality of $\mathcal{S}$. In particular, the constructed L-minimal surface is a Euclidean minimal surface.

The isotropic case: In this case we can let $S_{1}$ be the plane $S_{1}: x_{3}=-1$ with normal vector $(0,0,-1)$ and $S_{2}$ the sphere with signed radius $\frac{1}{2}$ and midpoint $\left(0,0, \frac{1}{2}\right)$. The top-view of the corresponding quadric $S_{i}^{2} \subset H_{i}$ is given by the elliptic paraboloid $\Sigma: x_{3}=\frac{1}{2}\left(x_{1}^{2}+x_{2}^{2}\right)$.

The pe-case: In the pe-case we can let $S_{1}=(0,0,-2,1)$ and $S_{2}=$ $(0,0,2,1)$. The top view $S_{M}$ of the corresponding quadric $S^{2}$ is given by the plane $x_{3}=0$. The hyperplane $H_{p e}$ is given as $\langle\mathbf{u}, \mathbf{x}\rangle+u_{0}=0$ with $\mathbf{u}=(0,0,1,0)$ and $u_{0}=0$ and the cyclographic image of the spheres in $H_{p e}$ consists of all spheres which are centered in a point of the plane $p_{H_{p e}}: x_{3}=0$.

We arrive at the following construction of discrete L-minimal surfaces that operates only in $E^{3}$ (see Figure 11):

1. Construct a Koebe mesh $\mathcal{S}$ tangent to a Euclidean sphere. 

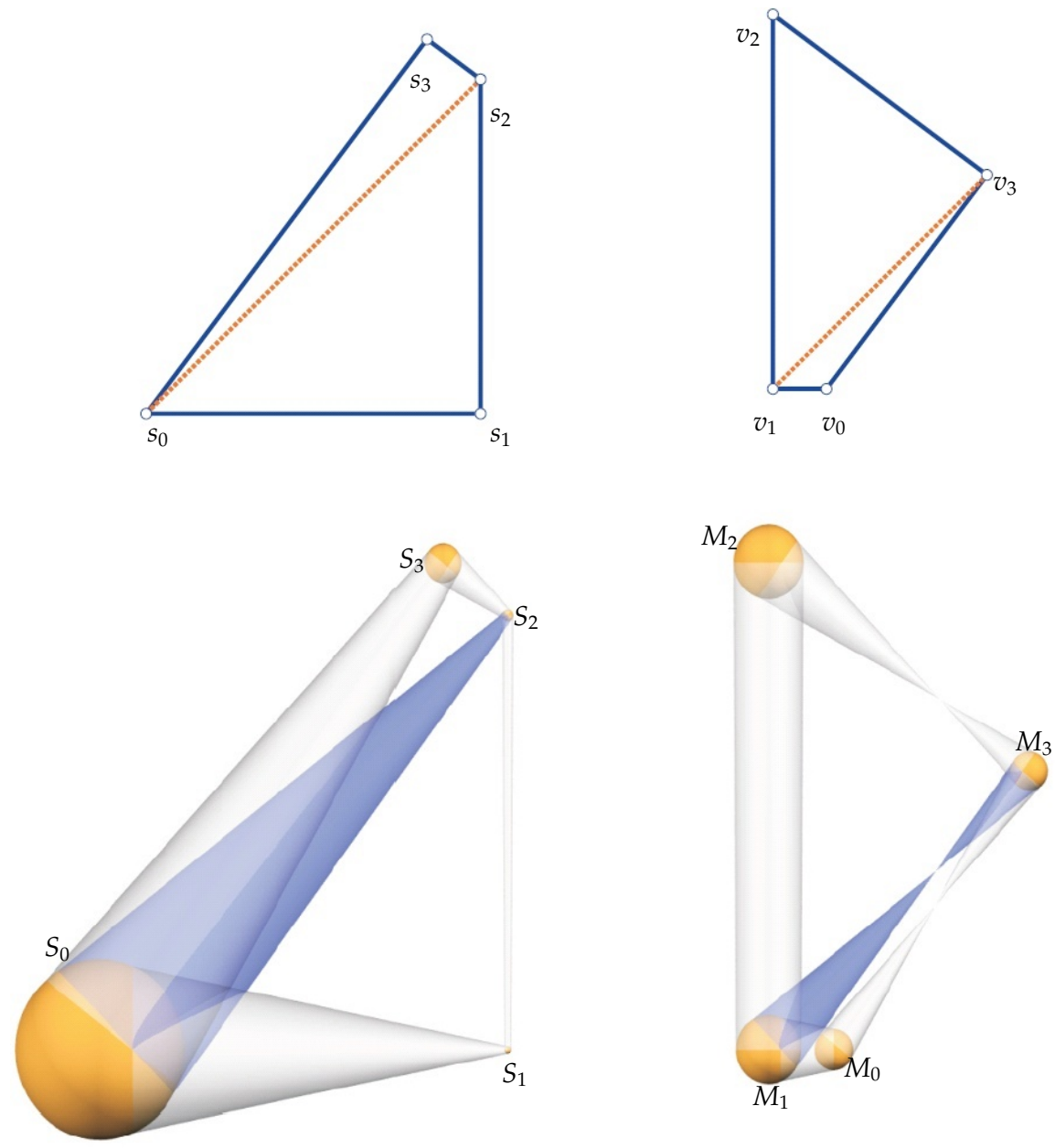

Fig. 10 Two parallel quads $\left[s_{0}, s_{1}, s_{2}, s_{3}\right],\left[v_{0}, v_{1}, v_{2}, v_{3}\right]$ with antiparallel diagonals are Christoffel transforms of each other (top). Two quadrilaterals of spheres $\left[S_{0}, S_{1}, S_{2}, S_{3}\right]$, $\left[M_{0}, M_{1}, M_{2}, M_{3}\right]$ are L-Christoffel transforms of each other if the edge cones are parallel and the diagonal cones are antiparallel (bottom).

2. $S$ yields three different Koebe pairs: A Euclidean one (which may be identified with $S$ itself), an isotropic one $\left(S_{1}^{i}, S_{2}^{i}\right)$ and a pe-Koebe pair $\left(S_{1}^{p e}, S_{2}^{p e}\right)$ (cf. Fig 12).

3. Applying the L-Christoffel dual construction to the vertex spheres of the three Koebe pairs yields the middle spheres of three different L-minimal surfaces.

4. Parallel translating the vertex cones of the three Koebe pairs gives us three different L-minimal surfaces modeled as EO-meshes.

Examples: Here we give a few examples of our construction of Lminimal EO-meshes. 

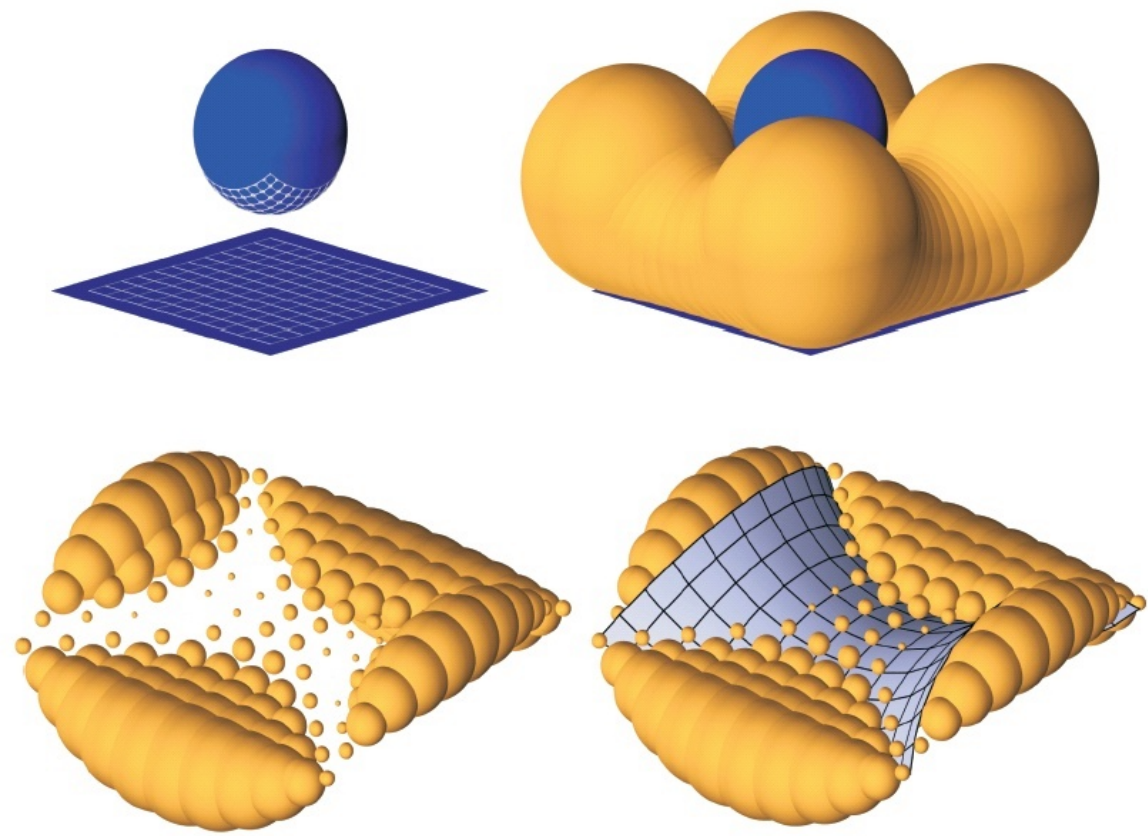

Fig. 11 General construction scheme of an L-minimal EO mesh: We start with a Koebe pair (top left). Next the middle spheres are computed (top right). After that the LChristoffel dual of the mesh consisting of the middle spheres is constructed (bottom left). The resulting spherical mesh consists of the middle spheres of the minimal surface. By parallel translating the vertex cones of $\mathcal{S}_{1}$ resp. $\mathcal{S}_{2}$ to the middle spheres, we arrive at the L-minimal EO mesh (bottom right). The second part of the L-minimal surface which is not shown here consists of a planar mesh, since the figure illustrates the construction of an L-minimal surface of the spherical type.
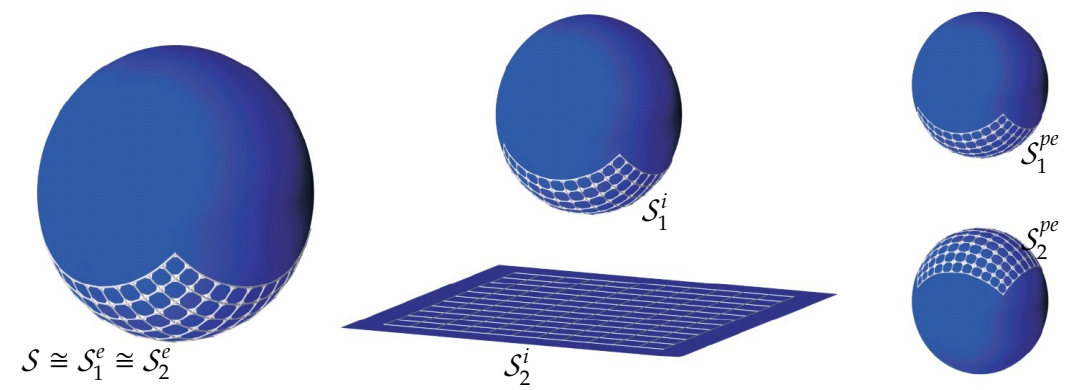

Fig. 12 A Koebe mesh $S$ (left) yields three different types of Koebe pairs: A Euclidean pair $\left(\mathcal{S}_{1}^{e}, \mathcal{S}_{2}^{e}\right)$ which can be identified with $\mathcal{S}$, an isotropic Koebe pair $\left(\mathcal{S}_{1}^{i}, \mathcal{S}_{2}^{i}\right)$ (middle) and a pe-Koebe pair $\left(\mathcal{S}_{1}^{p e}, \mathcal{S}_{2}^{p e}\right)$ (right).

Example 13 This example is constructed from a Koebe mesh of the type of an Enneper minimal surface (cf. Fig. 13). The minimal surfaces are shown in Figure 14. 

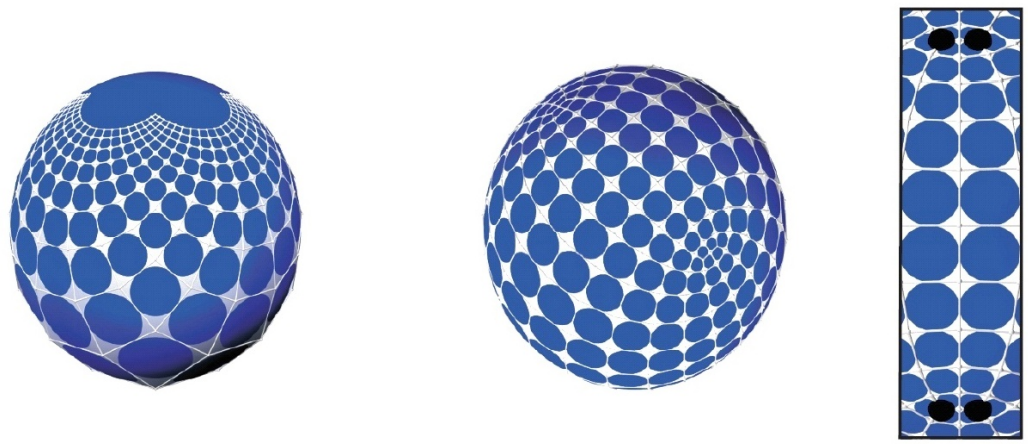

Fig. 13 Left: Koebe mesh of an Enneper surface. Middle: Koebe mesh of a Scherk surface. Right: This mesh contains eight irregular vertices with valence 3 (only four of them can be seen in the picture). The correct interpretation of this Koebe mesh is to view it as branched covering of the sphere with the irregular vertices as branch points.

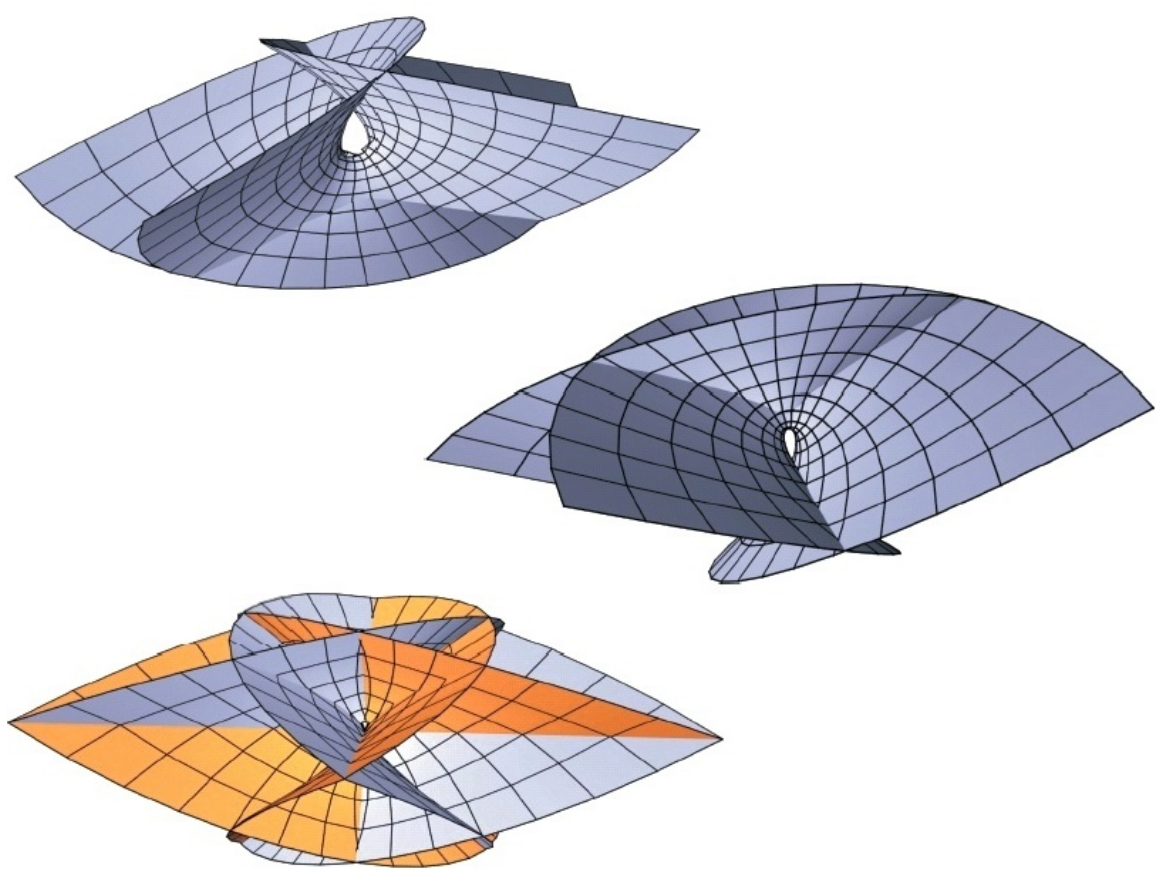

Fig. 14 L-minimal EO meshes generated from an Enneper Koebe mesh: The discrete Euclidean Enneper surface is depicted at the top. In the middle we see the corresponding minimal surface of the spherical type, and at the bottom we see the Bonnet minimal surface. Again, the Bonnet surface consists of two sheets.

Example 14 Our next example is constructed from a Koebe mesh of the type of a Scherk minimal surface (cf. Fig. 13). Minimal surfaces are shown in Figure 15. This example is somewhat more complicated than the Enneper case since the combinatorics is not regular. There are eight points of valence three which 


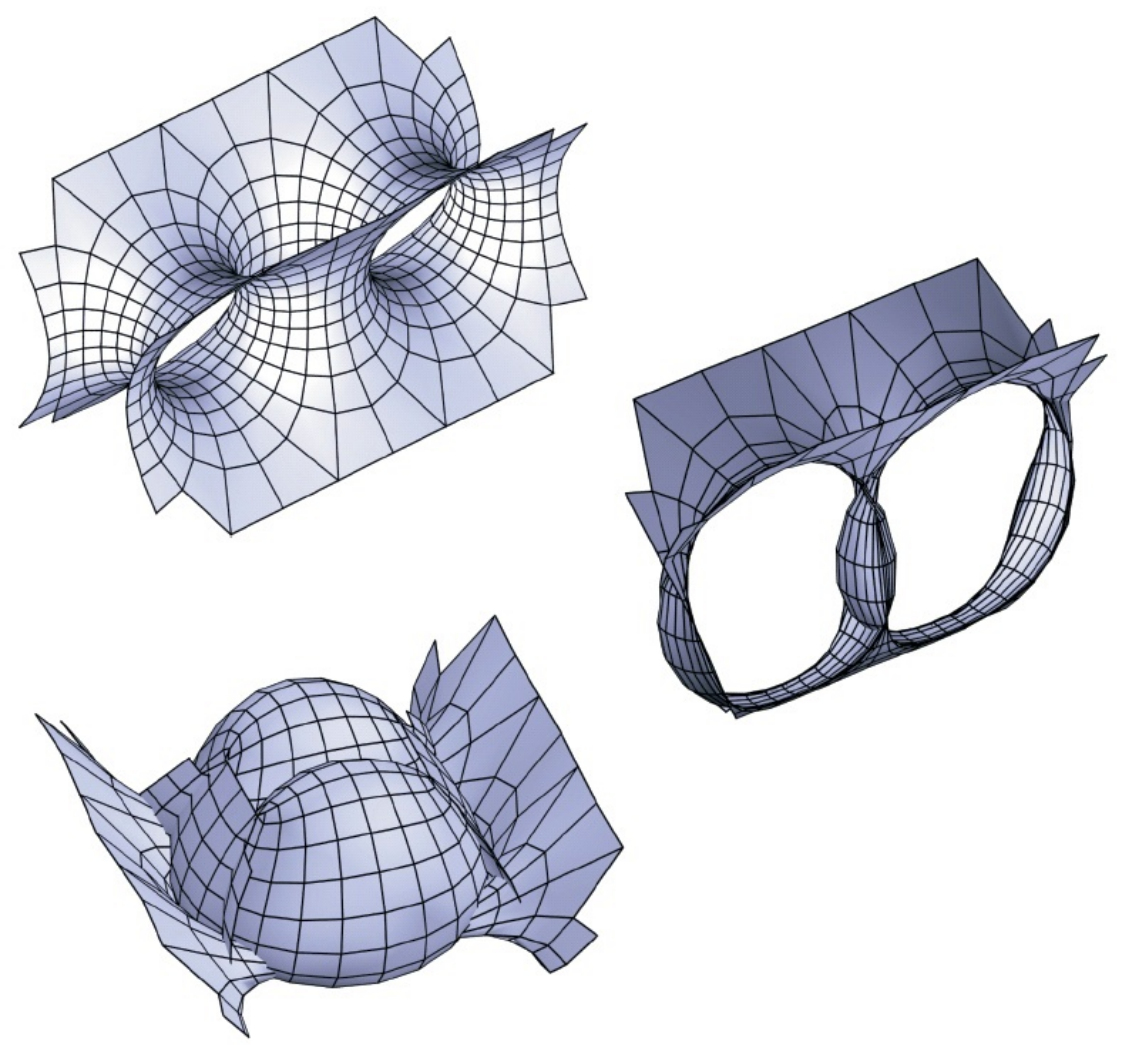

Fig. 15 L-minimal EO meshes generated from an Scherk Koebe mesh: The discrete Euclidean Scherk surface is depicted at the top. In the middle we see the corresponding minimal surface of the spherical type and at the bottom the minimal surface of Bonnet type. For visual reasons only one sheet is shown.

in the continuous setting correspond to branch points with branch number one (see [BHS06]). When carrying out the Christoffel dual construction, the minimal surface ramifies around these points and therefore the correct interpretation of the Koebe mesh corresponding to the Scherk minimal surface is to view it as a branched covering of the unit sphere. Note that the Scherk minimal surfaces of the spherical and Bonnet type possess a considerable number of cusps and self-intersections - a common phenomenon for L-minimal surfaces [PGM08].

\section{Conclusion and future work}

Using the classical approach to Laguerre geometry via the cyclographic mapping, we have proved the invariance of EO meshes under Laguerre transformations. Generalizing this result, we could show that EO meshes $\mathcal{M}$ in hyperplanes of Minkowski 4-space are cyclographically projected to 
pairs of Euclidean EO meshes. Application of this procedure to a minimal mesh $\mathcal{M}$ results in those EO meshes which can also be viewed as discrete Laguerre-minimal surfaces. We have also provided a direct construction of L-minimal EO meshes with help of a Laguerre geometric variant of Christoffel duality, applied to certain pairs of Koebe meshes.

Among the many directions for future research, we would like to highlight the following ones:

1. The characterization of L-minimal surface can be based on the concept of middle spheres [Bla29]. While these spheres appeared in our study, we are still lacking a general discrete definition of middle spheres for meshes which are objects of Laguerre geometry (conical meshes and EO meshes).

2. The problem of constructing general discrete Laguerre-minimal surfaces remains open. Especially in view of the results in [PGM08], it can be conjectured that this construction is slightly simpler than the also unknown construction of discrete Willmore surfaces.

3. EO meshes are discrete versions of Laguerre isothermic surfaces (cf. [PLW*07]). Especially for applications in architectural design it would be highly interesting to get more insight into the possible shapes of $\mathrm{La}$ guerre isothermic surfaces. While this may be a too difficult task, it would already be helpful to know about important shape restrictions one has to deal with when designing with quadrilateral EO meshes.

4. For practical design with EO meshes or conical meshes, it may be interesting to provide an intuitive interactive way of modifying a mesh with help of Laguerre-transformations.

\section{Acknowledgments}

This research was supported by grants No. 19214-N18, S92-N12 of the Austrian Science Fund (FWF) and by Project MLFS which is funded by FFG and Waagner Biro Stahlbau AG. We thank Stefan Sechelmann for providing us with the Scherk Koebe polyhedron in Figure 13 (right) and Heinz Schmiedhofer for creating the design in Figure 1.

\section{References}

BHS06. Bobenko A., Hoffmann T., Springborn B. A.: Minimal surfaces from circle patterns: Geometry from combinatorics. Ann. of Math. 164 (2006), 231-264.

Bla24. BlaschKe W.: Über die Geometrie von Laguerre II: Flächentheorie in Ebenenkoordinaten. Abh. Math. Sem. Univ. Hamburg 3 (1924), 195-212.

Bla25. BlaschKe W.: Über die Geometrie von Laguerre III: Beiträge zur Flächentheorie. Abh. Math. Sem. Univ. Hamburg 4 (1925), 1-12.

Bla29. Blaschкe W.: Vorlesungen über Differentialgeometrie, vol. 3. Springer, 1929.

BS04. Bobenko A. I., Springborn B.: Variational principles for circle patterns and Koebe's theorem. Trans. Amer. Math. Soc. 356 (2004), 659-689. 
BS06. Bobenko A., Suris Yu.: On organizing principles of discrete differential geometry, geometry of spheres. to appear. http://arxiv.org/abs/math.DG/ 0608291, 2006.

BS08. Bobenko A., Suris Yu.: Discrete differential geometry. Integrability. Graduate Studies in Mathematics. AMS, 2008.

Cec92. CECIL T.: Lie Sphere Geometry. Springer, 1992.

G*02. Glymph J., Eт AL.: A parametric strategy for freeform glass structures using quadrilateral planar facets. In Acadia 2002 (2002), ACM, pp. 303-321.

Kön26. KöNig K.: L-Minimalflächen. Mitt. Math. Ges. Hamburg (1926), 189-203.

Kön28. König K.: L-Minimalflächen II. Mitt. Math. Ges. Hamburg (1928), 378-382.

LPW*06. Liu Y., Pottmann H., Wallner J., YANG Y.-L., WANG W.: Geometric modeling with conical meshes and developable surfaces. ACM Trans. Graphics 25, 3 (2006), 681-689.

MN95. Musso E., Nicolodi L.: L-minimal canal surfaces. Rendiconti di Mat. 15 (1995), 421-445.

MN96. Musso E., Nicolodi L.: A variational problem for surfaces in Laguerre geometry. Trans. Amer. Math. Soc. 348 (1996), 4321-4337.

PAHK07. Pottmann H., Asperl A., Hofer M., Kilian A.: Architectural Geometry. Bentley Institute Press, 2007.

Pa199. Palmer B.: Remarks on a variational problem in Laguerre geometry. Rendiconti di Mat. 19 (1999), 281-293.

PCW07. Рottmann H., Cokcan S. B., Wallner J.: Discrete surfaces for architectural design. In Curves and Surfaces: Avignon 2006, Lyche T., Merrien J. L., Schumaker L. L., (Eds.). Nashboro Press, 2007, pp. 213-234.

PGM08. Pottmann H., Grohs P., Mitra N. J.: Laguerre minimal surfaces, isotropic geometry and linear elasticity. Adv. Comp. Math (2008). to appear.

PL07. Pottmann H., Liu Y.: Discrete surfaces of isotropic geometry with applications in architecture. In The Mathematics of Surfaces, Martin R., Sabin M., Winkler J., (Eds.). Springer, 2007, pp. 341-363. Lecture Notes in Computer Science 4647.

PLW*07. Pottmann H., Liu Y., Wallner J., Bobenko A., Wang W.: Geometry of multi-layer freeform structures for architecture. ACM Trans. Graphics 26, 3 (2007), \#65,1-11.

PP98. Pottmann H., Peternell M.: Applications of Laguerre geometry in CAGD. Comp. Aid. Geom. Des. 15 (1998), 165-186.

PW07. Pottmann H., Wallner J.: The focal geometry of circular and conical meshes. Adv. Comput. Math (2007). to appear.

Sch97. Sснramm O.: Circle patterns with the combinatorics of the square grid. Duke Math. J. 86 (1997), 347-389.

Sch03. SсновеR H.: Freeform glass structures. In Glass Processing Days 2003. Glass Processing Days, Tampere (Fin.), 2003, pp. 46-50.

WP08. Wallner J., Pottmann H.: Infinitesimally flexible meshes and discrete minimal surfaces. Monatshefte Math. 153, 347-365 (2008).

Zie95. Ziegler G.: Lectures on Polytopes. Springer, 1995. 